\title{
Review on Sublethal Effects of Environmental Contaminants in Honey Bees (Apis mellifera), Knowledge Gaps and Future Perspectives
}

\author{
Agata Di Noi $^{1}{ }^{1}$, Silvia Casini $^{2, *}$, Tommaso Campani ${ }^{2}{ }^{\circledR}$, Giampiero Cai $^{1}{ }^{\circledR}$ and Ilaria Caliani ${ }^{2}$ \\ 1 Department of Life Sciences, University of Siena, via Mattioli, 4, 53100 Siena, Italy; \\ agata.dinoi@student.unisi.it (A.D.N.); cai@unisi.it (G.C.) \\ 2 Department of Physical, Earth and Environmental Sciences, University of Siena, via Mattioli, 4, \\ 53100 Siena, Italy; campani@unisi.it (T.C.); caliani4@unisi.it (I.C.) \\ * Correspondence: silvia.casini@unisi.it
}

check for updates

Citation: Di Noi, A.; Casini, S.; Campani, T.; Cai, G.; Caliani, I. Review on Sublethal Effects of Environmental Contaminants in Honey Bees (Apis mellifera), Knowledge Gaps and Future Perspectives. Int. J. Environ. Res. Public Health 2021, 18, 1863. https:// doi.org/10.3390/ijerph18041863

Academic Editor:

Alexandra Schneider

Received: 31 December 2020

Accepted: 10 February 2021

Published: 14 February 2021

Publisher's Note: MDPI stays neutral with regard to jurisdictional claims in published maps and institutional affiliations.

Copyright: (c) 2021 by the authors. Licensee MDPI, Basel, Switzerland. This article is an open access article distributed under the terms and conditions of the Creative Commons Attribution (CC BY) license (https:// creativecommons.org/licenses/by/ $4.0 /)$.

\begin{abstract}
Honey bees and the pollination services they provide are fundamental for agriculture and biodiversity. Agrochemical products and other classes of contaminants, such as trace elements and polycyclic aromatic hydrocarbons, contribute to the general decline of bees' populations. For this reason, effects, and particularly sublethal effects of contaminants need to be investigated. We conducted a review of the existing literature regarding the type of effects evaluated in Apis mellifera, collecting information about regions, methodological approaches, the type of contaminants, and honey bees' life stages. Europe and North America are the regions in which A. mellifera biological responses were mostly studied and the most investigated compounds are insecticides. A. mellifera was studied more in the laboratory than in field conditions. Through the observation of the different responses examined, we found that there were several knowledge gaps that should be addressed, particularly within enzymatic and molecular responses, such as those regarding the immune system and genotoxicity. The importance of developing an integrated approach that combines responses at different levels, from molecular to organism and population, needs to be highlighted in order to evaluate the impact of anthropogenic contamination on this pollinator species.
\end{abstract}

Keywords: honey bees; sublethal effects; plant protection products; bees decline; monitoring strategies; methodological approach

\section{Introduction}

Honey bees (Apis mellifera) are essential organisms for the environment, in particular for their critical roles in the pollination of crops, flowers, and fruit trees [1-3]. It has been estimated that honey bees are responsible for providing a pollination service to $96 \%$ of animal-pollinated crops $[4,5]$. Bees are also indirectly responsible for the reproduction and maintenance of wild plant communities and biodiversity [6-8]. Their value to global food crops is estimated at $€ 153$ billion per year [9]. In addition, honey bees provide honey, pollen, wax, propolis, and royal jelly to humans [10]. Throughout the last decade, declines in bees and other pollinators have been observed globally [11-13]; important honey bee colony losses have been reported, particularly in North America and Western Europe [14-16]. It was beekeepers who alerted the scientific community of this vital colony mortality, since they monitor bee colonies worldwide and are immediately aware of any kind of changes to the bees' colony [17]. This decline has led to concerns over there being a sustainable food supply and the health of natural ecosystems [18]. The causes of pollinator decline may be complex and subject to disagreement. However, the general weakening and death of bee colonies has been observed to be mainly caused by the combined effects of multiple stressors [3,19-21], such as biological factors [22,23], environmental factors [19,24,25], chemical and nutritional stressors [26,27], chemical and biological factors [28-33] and multiple 
chemicals [34-36]. In particular, this last kind of stressor is a matter of great concern since bees can be exposed to a wide range of chemical mixtures, including anthropogenic compounds, such as plant protection products (PPPs) or veterinary drugs, and those of natural origin, such as mycotoxins, flavonoids and plant toxins [20,37,38]. Although PPPs, such as insecticides, acaricides, herbicides, and fungicides, have many benefits for agriculture [39], there are also several potential risks associated with their use, such as pest resistance, resurgence, and secondary pest outbreaks, as well as wider environmental contamination and human health concerns [40-42]. Although insecticides are applied to target insect pests, their use in agriculture can affect non-target insects that provide beneficial services to agriculture. Among these beneficial insects, the focus was on social bees, with a particular interest in neonicotinoid insecticides and their lethal and sublethal effects at colony and population levels. Nonetheless, other PPPs used in modern agriculture, such as fungicides and herbicides, were demonstrated to affect honey bee's health status [43-46].

The sublethal effects of PPPs and other anthropogenic contaminants in Apis mellifera need to be investigated. A wide range of studies investigated mortality and accumulation in honey bees, in order to obtain data related to contamination that may affect these organisms [33,47-49]. Moreover, studies concerning the general fitness of honey bees, which examined their behaviour, flight activity, and sensory ability, were conducted over the years to observe the macroscopic effects of contaminants [48,50-52]. To a lesser extent, enzymatic and molecular responses have also been studied, using genomic, metabolomic, and transcriptomic techniques and biomarkers $[43,53-56]$, in order to increase understanding of the anthropogenic impact on these insects.

The current manuscript aims to provide a review of the available toxicological studies about the biological responses of honey bees to external stressors. In particular, we focused on where studies were carried out, we examined the contaminants involved, methodological approaches, honey bees' life stages, and the different kind of responses considered in each paper, with the purpose to determine and identify knowledge gaps. This review could also provide indications regarding possible improvements in the monitoring approach, both in a scientific and regulatory way.

\section{Materials and Methods}

The search for scientific papers was conducted on ScienceDirect, Google Scholar, and One search database, using the following search terms to find relevant literature: "Apis mellifera", "honey bees", "biomarkers", "ecotoxicology", "toxicology", "sublethal effects", and "biochemical analysis". To extend the collection of the relevant literature, the bibliographical references of each article were also examined. The selected articles were written in English and the full text version is available. Grey literature and non-accessible peer-reviewed articles were not included in our work, and this resulted in a primary dataset of 846 publications.

Papers considered for this review included investigations into toxicity effects, sublethal behavioural effects, impacts on bees at a genetic, molecular, or physiological level. Studies that reported only LC50 and LD50 were omitted from our analysis. The final dataset included a total of 106 research papers. For each paper, we extracted the following information: a complete bibliographical reference, a methodological approach, the investigated compounds, the life stage, and the studied responses. Where multiple categories of any variable were reported in the same paper, all were included in the final analyses. Methodological approaches were divided into three categories: "laboratory", "semi-field" and "field". "Laboratory" studies were defined as those carried out within the laboratory, with the exposure of honey bees to contaminants. "Semi-field" studies were defined as those that were conducted outdoors, but confined to bees, e.g., using exclusion cages. "Field" studies were defined as studies conducted outdoors with no restriction on the bees' movements and the data were collected in the field. 
The compounds studied in the papers were divided into insecticides, herbicides, fungicides, acaricides, trace elements, polycyclic aromatic hydrocarbons (PAHs), parasites, radioactivity, mixtures, and other compounds.

The following life stages were considered: "Brood", "Pupae", "Larvae", "Adults" and "Queens". If the life stages at which bees were exposed to pesticides differed from the life stage at which the effects were measured, then both were included in the final analyses.

Examining the existing literature, we described fifteen different "effect types" that were assessed, including morphology, apoptosis and necrosis, histopathology, cytotoxicity, consumption, foraging activity, and fitness, learning ability, other behaviours, physiological function and morphology, reproduction, sensory (gustatory or olfactory), flight activity, growth and development and, accumulation. Research studies were placed into multiple categories if they contained more than one effect type.

Moreover, we isolated more specific responses, mostly characterized by biomarkers and transcriptomic, metabolomic, proteomic approaches, in nine endpoints: detoxification, neurotoxicity, immunity, metabolism, oxidative stress, genotoxicity, primary stress response, carbohydrates assay, and protein amounts. Where studies included more than one option in any of the variables measured, it was included in analyses of both.

\section{Results}

\subsection{Where Studies Took Place}

Most studies examined for this review were carried out in Europe (48) and North America (35), followed by Asia (11) and South America (9), Africa (8) and Australia (3) (Figure 1).

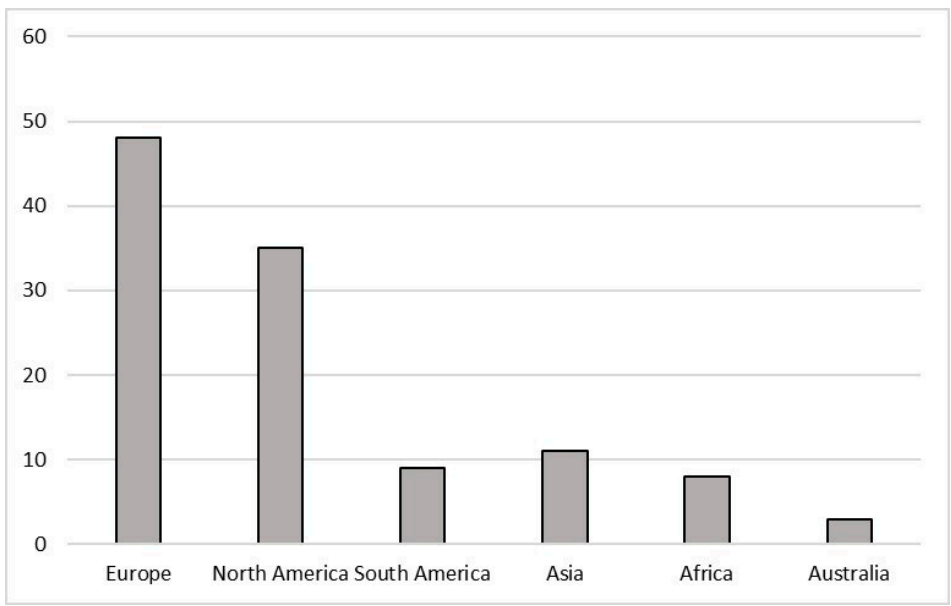

Figure 1. Number of studies, conducted on Apis mellifera, and divided by continent, that met the criteria for inclusion in this review.

\subsection{Methodological Approaches}

As shown in Figure 2, most studies were carried out under laboratory conditions (63), with 14 studies carried out in semi-field conditions, and 25 at the full field scale.

\subsection{Life Stages}

The bibliographical research highlighted that most of the studies, as shown in Figure 3, were conducted on adult bees (99), followed by larvae (9), brood (7), and pupae stage (4). Only 2 studies, that met the criteria of this work, were about queen bees.

\subsection{Studied Compounds}

Insecticides were investigated in 71 studies, followed by trace elements, in 15 papers. Studies on acaricides (12), herbicides (12), and fungicides (11) were present with a similar number. Mixtures and PAHs are still poorly studied, respectively with 8 and 2 papers 
(Figure 4). In the "other compounds" category, $\mathrm{SO}_{2}$, ethyl methane-sulfonate (EMS), ethanol and pharmaceutical compounds were included. In the category "parasites" are present not only papers that examined reactions to parasites but also other contaminants; there are not any papers that studied only parasites since they did not satisfy the criteria used for this review.

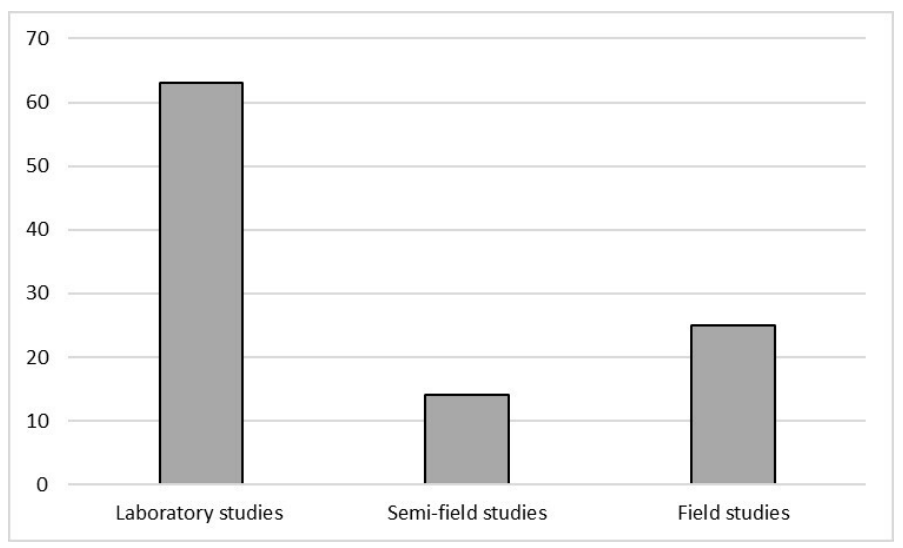

Figure 2. Number of studies, divided by a methodological approach, on Apis mellifera, that met the criteria for inclusion in this review.

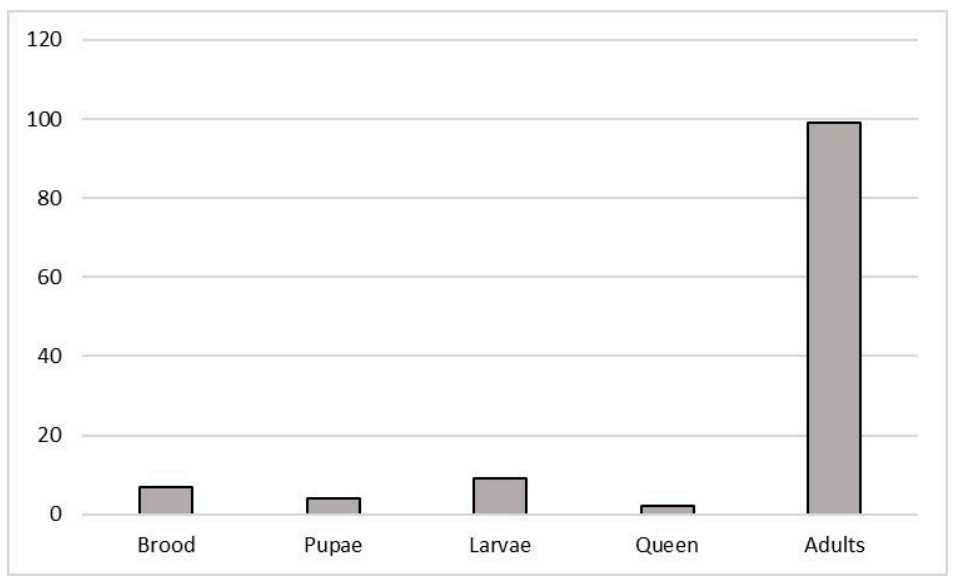

Figure 3. Number of studies, divided by life stages, on Apis mellifera, that met the criteria for inclusion in this review.

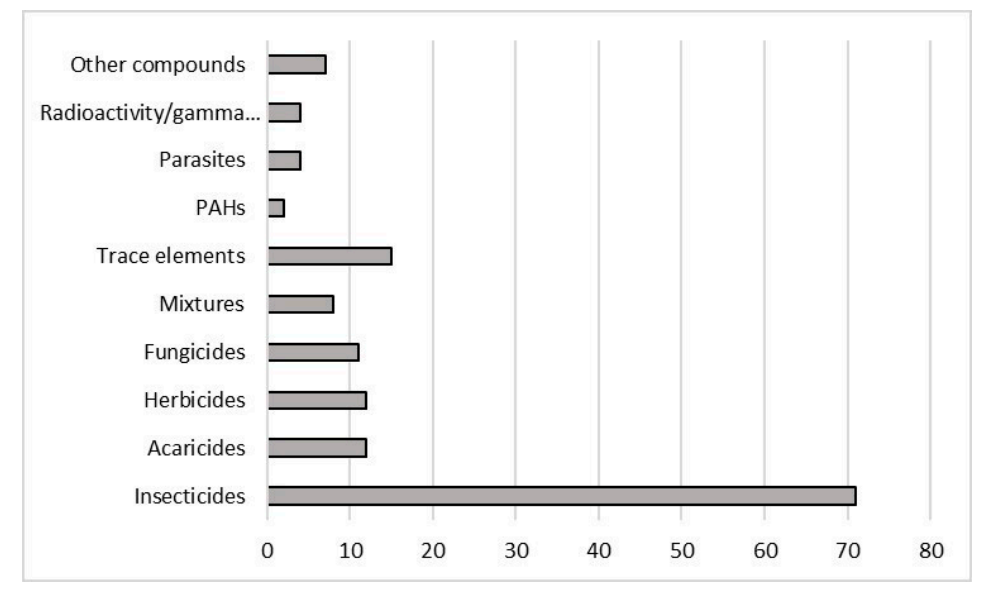

Figure 4. Number of studies, divided by kind of compounds, on Apis mellifera, that met the criteria for inclusion in this review. 


\subsection{Effect Type}

Most studies used for this review investigated more than one effect (64 studies) on honey bees but 42 studies concentrated on investigating just one effect. The most widely studied single effect type was accumulation (20) followed by foraging activity (15) studies (Figure 5). Figure 6 shows studies regarding enzymatic and molecular responses (58): the effects that were studied in more depth were detoxification (27) and neurotoxicity (26), followed by metabolic responses (21), immunity (17), and oxidative stress (15).

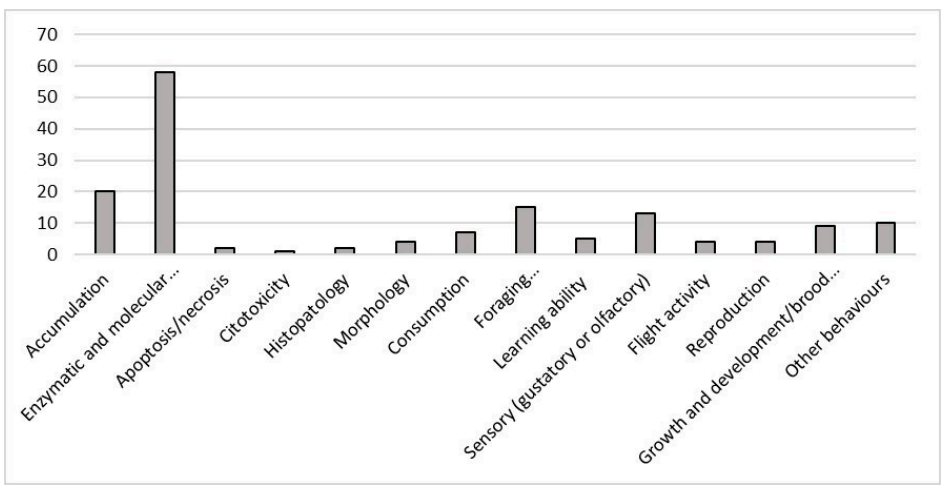

Figure 5. Number of studies, divided by kind of responses, on Apis mellifera, that met the criteria for inclusion in this review.

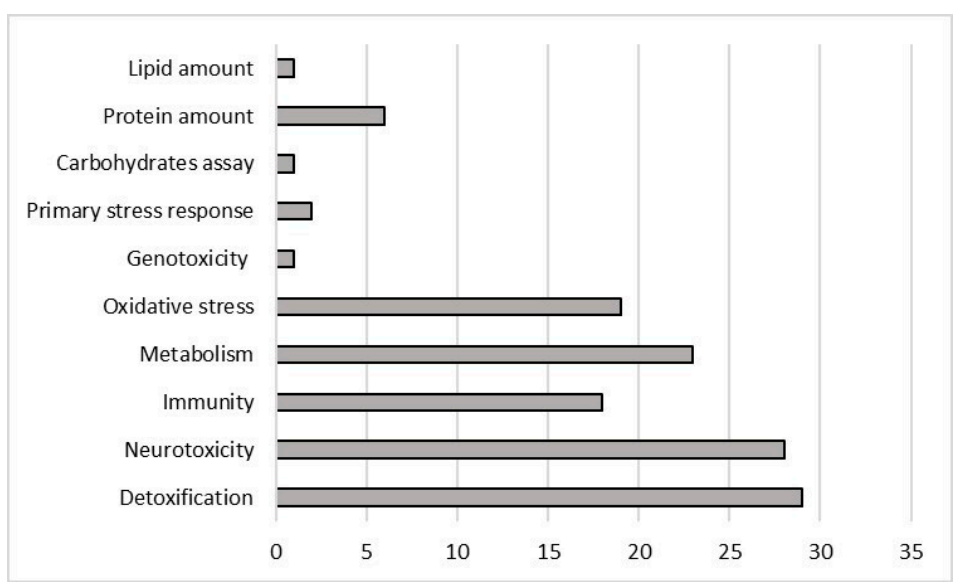

Figure 6. Number of studies, divided by molecular and enzymatic responses, on Apis mellifera, that met the criteria for inclusion in this review.

In the following tables, all the examined papers are summarized by endpoint; there are two tables for each methodological approach, one for cellular to whole organism and population endpoints, and one for molecular and enzymatic endpoints.

Endpoints examined in laboratory studies are summarized in Tables 1 and 2. Table 1 shows two endpoints were most used in laboratory studies, "foraging activity/fitness/ production of matrixes" and "sensory (gustatory or olfactory)", both with a total of 12 papers.

Table 2 shows the molecular and enzymatic endpoints examined in laboratory studies. The most studied effect concerned "neurotoxicity" (24 studies) and the test that was applied most frequently was the acetylcholinesterase (AChE) activity; only two papers examined the presence of trembling, hyperactivity, and paralysis in the organisms exposed mostly to insecticides. The second most investigated endpoint was "detoxification", with studies mostly concerning the activity of glutathione-S-transferase (GST) or CYP450. Another endpoint with a considerable number of papers (17) was "metabolism", in which alkaline phosphatase (ALP) and ATPase were mostly examined. "Oxidative stress" endpoint was 
examined only in 14 papers, evaluating the activity of antioxidant enzymes such as catalase (CAT) and superoxide dismutase (SOD).

In semi-field studies, the most frequently studied endpoints are "foraging activity/fitness/production of matrixes" and "other behaviors", both with 6 studies (Table 3). In Table 4 the molecular endpoints are summarized; in this case the most examined endpoints ( 3 studies) were "protein amount" and "immunity", followed by "detoxification", with 2 papers.

The endpoints examined in field studies are summarized in Tables 5 and 6 . Table 5 shows that 16 studies observed "accumulation" in the honey bees sampled in sites with different levels of anthropogenic pressure. In general, herbicides and insecticides were the contaminants that tended to be observed more in these accumulation studies.

Table 1. Summary of laboratory studies divided by endpoint and contaminants.

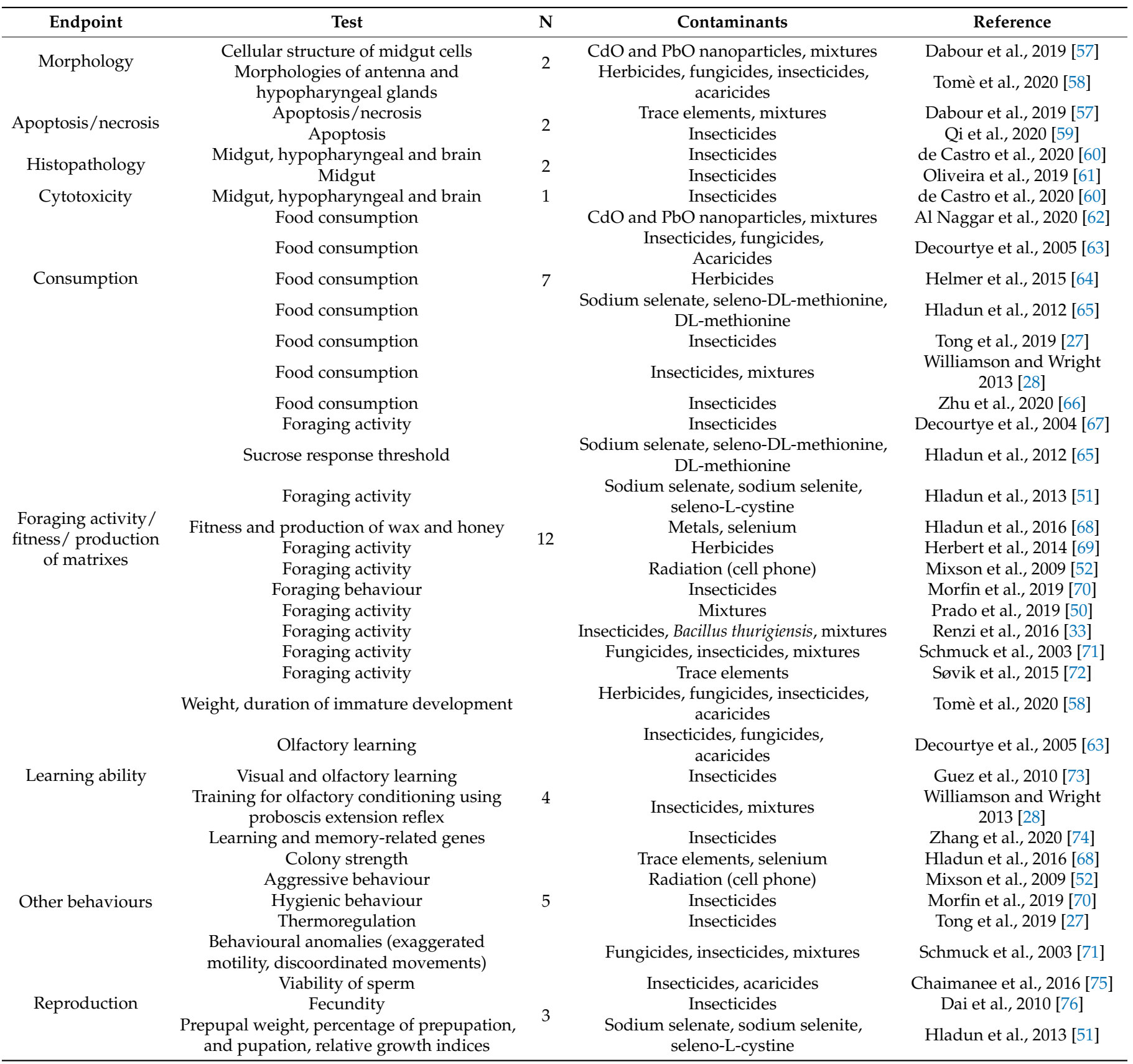


Table 1. Cont.

\begin{tabular}{|c|c|c|c|c|}
\hline Endpoint & Test & $\mathbf{N}$ & Contaminants & Reference \\
\hline \multirow{12}{*}{$\begin{array}{l}\text { Sensory (gustatory } \\
\text { or olfactory) }\end{array}$} & $\begin{array}{l}\text { Olfactory conditioning of Proboscis } \\
\text { extension reflex (PER) }\end{array}$ & \multirow{12}{*}{12} & Insecticides & Al Naggar et al., 2015 [77] \\
\hline & PER & & Insecticides, acaricides & Decourtye et al., 2004 [67] \\
\hline & PER & & $\begin{array}{l}\text { Insecticides, fungicides, } \\
\text { acaricides }\end{array}$ & Decourtye et al., 2005 [63] \\
\hline & PER & & Insecticides & Guez et al., 2010 [73] \\
\hline & $\begin{array}{c}\text { Antennal response assays, Proboscis } \\
\text { response assays }\end{array}$ & & $\begin{array}{l}\text { Sodium selenate, seleno-DL-methionine, } \\
\text { DL-methionine }\end{array}$ & Hladun et al., 2012 [65] \\
\hline & PER & & Herbicides & Herbert et al., 2014 [69] \\
\hline & PER & & Insecticides & Imran et al., 2019 [78] \\
\hline & PER & & Radiation (cell phone) & Mixson et al., 2009 [52] \\
\hline & PER & & Insecticides, acaricides & Weick and Thorn 2002 [79] \\
\hline & PER & & Insecticides, mixtures & $\begin{array}{l}\text { Williamson and Wright } \\
2013[28]\end{array}$ \\
\hline & PER & & Insecticides & Wright et al., 2015 [80] \\
\hline & PER & & Insecticides & Yang et al., 2012 [81] \\
\hline \multirow{4}{*}{ Flight activity } & Flight navigation & \multirow{3}{*}{3} & Radiation (cell phone) & Mixson et al., 2009 [52] \\
\hline & Flight ability and success & & Insecticides & Tong et al., 2019 [27] \\
\hline & Flight activity & & Mixtures & Prado et al., 2019 [50] \\
\hline & Growth of adult workers & \multirow{5}{*}{5} & Insecticides, Varroa destructor & Abbo et al., 2017 [47] \\
\hline \multirow{4}{*}{$\begin{array}{l}\text { Growth and devel- } \\
\text { opment/brood } \\
\text { production }\end{array}$} & Growth and development & & Insecticides & Dai et al., 2010 [76] \\
\hline & Larval growth and development & & Insecticides & du Rand et al., 2017 [82] \\
\hline & Brood production & & Trace elements, selenium & Hladun et al., 2016 [68] \\
\hline & Duration of immature development & & $\begin{array}{l}\text { Herbicides, fungicides, insecticides, } \\
\text { acaricides }\end{array}$ & Tomè et al., 2020 [58] \\
\hline \multirow{2}{*}{ Accumulation } & Chemical analysis & \multirow{2}{*}{2} & $\begin{array}{l}\text { Sodium selenate, sodium selenite, } \\
\text { seleno-L-cystine }\end{array}$ & Hladun et al., 2013 [51] \\
\hline & Chemical analysis & & Trace elements, selenium & Hladun et al., 2016 [68] \\
\hline
\end{tabular}

Table 2. Summary of laboratory studies divided by molecular and enzymatic endpoint and contaminants.

\begin{tabular}{|c|c|c|c|c|}
\hline Endpoint & Test & $\mathbf{n}$ & Contaminants & Reference \\
\hline \multirow{23}{*}{ Detoxification } & $\begin{array}{c}\text { CYP genes expression, } \\
\text { glutathione-S-transferase (GST) genes } \\
\text { expression }\end{array}$ & \multirow{23}{*}{23} & \multirow{3}{*}{$\begin{array}{l}\mathrm{CdO} \text { and } \mathrm{PbO} \text { nanoparticles, mixtures } \\
\text { Insecticides, fungicides, herbicides and } \\
\text { mixture }\end{array}$} & Al Naggar et al., 2015 [77] \\
\hline & CYP and GST genes expression & & & Al Naggar et al., 2020 [62] \\
\hline & (GST) & & & Almasri et al., 2020 [83] \\
\hline & GST & & Insecticides & Badawy et al., 2015 [84] \\
\hline & GST and CaEs & & Insecticides & Badiou-Bénéteau et al., 2012 [53] \\
\hline & GST and $\mathrm{CaE}$ & & Fungicides, metals, EMS & Caliani et al., 2021 [43] \\
\hline & GST & & Insecticides & Carvalho et al., 2013 [55] \\
\hline & Detoxification genes expression & & Insecticides, acaricides & Chaimanee et al., 2016 [75] \\
\hline & $\begin{array}{c}\text { Genes encoding CYP } 450 \\
\text { monooxygenases }\end{array}$ & & Insecticides & Christen et al., 2019 [85] \\
\hline & $\begin{array}{l}\text { Genes encoding CYP450 } \\
\text { monooxygenases }\end{array}$ & & Insecticides & Christen et al., 2019 [86] \\
\hline & Proteomic and metabolomic analysis & & \multirow{2}{*}{$\begin{array}{c}\text { Insecticides } \\
\text { Herbicides, fungicides, insecticides, } \\
\text { Varroa destructor }\end{array}$} & du Rand et al., 2017 [82] \\
\hline & Detoxification genes expression & & & Gregorc et al., 2012 [87] \\
\hline & $\begin{array}{c}\text { cytochrome P450 (CYP450), GST and } \\
\text { CaEs }\end{array}$ & & \multirow{8}{*}{$\begin{array}{c}\text { Insecticides } \\
\text { Insecticides, Bacillus thurigiensis, mixtures } \\
\text { Herbicides, fungicides, insecticides, } \\
\text { acaricides }\end{array}$} & Johnson et al., 2006 [88] \\
\hline & CYP450 & & & Johnson et al., 2009 [89] \\
\hline & GST and $\mathrm{CaE}$ & & & Li et al., 2017 [90] \\
\hline & P450 genes expression & & & Mao et al., 2011 [91] \\
\hline & GST isoenzymes expression & & & Papadopoulos et al., 2004 [92] \\
\hline & GST, GR and gene expressions & & & Qi et al., 2020 [59] \\
\hline & GST & & & Renzi et al., 2016 [33] \\
\hline & P450 genes expression & & & Tomè et al., 2020 [58] \\
\hline & $\begin{array}{l}\text { Esterase (EST), GST, CYP450. CYPs and } \\
\text { GSTs transcript levels }\end{array}$ & & Insecticide & Yao et al., 2018 [93] \\
\hline & CYP450 and phospholipase A2 & & Insecticides & Zaworra and Nauen 2019 [94] \\
\hline & Detoxification genes expression & & Insecticides & Zhu et al., 2020 [66] \\
\hline
\end{tabular}


Table 2. Cont

\begin{tabular}{|c|c|c|c|c|}
\hline Endpoint & Test & $\mathbf{n}$ & Contaminants & Reference \\
\hline \multirow{27}{*}{ Neurotoxicity } & acetylcholinesterase (AChE) & \multirow{27}{*}{24} & Insecticides & Al Naggar et al., 2015 [77] \\
\hline & $\mathrm{AChE}$ & & & Al Naggar et al., 2020 \\
\hline & $\mathrm{AChE}$ and $\mathrm{CaE}-3$ & & $\begin{array}{l}\text { Insecticides, fungicides, herbicides and } \\
\text { mixture }\end{array}$ & Almasri et al., 2020 [83] \\
\hline & $\mathrm{AChE}$ & & Insecticides & Badawy et al., 2015 [84] \\
\hline & AChE & & Acaricides, mixtures & Badiou et al., 2008 [95] \\
\hline & AChE and CaEs & & Insecticides & Badiou-Bénéteau et al., 2012 [53] \\
\hline & $\mathrm{AChE}$ & & Insecticides & Bendahou et al., 1999 [96] \\
\hline & AChE & & Herbicides, insecticides & Boily et al., 2013 [97] \\
\hline & $\mathrm{AChE}$ and $\mathrm{CaE}$ & & Fungicides, trace elements, EMS & Caliani et al., 2021 [43] \\
\hline & $\mathrm{AChE}$ and $\mathrm{CaEs}$ & & Insecticides & Carvalho et al., 2013 [55] \\
\hline & Genes encoding acetylcholine receptors & & Insecticides & Christen et al., 2019 [85] \\
\hline & Genes encoding acetylcholine receptors & & Insecticides & Christen et al., 2019 [86] \\
\hline & Trembling and paralysis & & Insecticides, acaricides & Decourtye et al., 2004 [67] \\
\hline & $\mathrm{AChE}$ and $\mathrm{CaEs}$ & & Gamma irradiation & Gagnaire et al., 2019 [98] \\
\hline & $\mathrm{AChE}$ & & Insecticides & Glavan et al., 2018 [99] \\
\hline & Esterase & & Insecticides & Hashimoto et al., 2003 [100] \\
\hline & $\mathrm{AChE}$ and $\mathrm{CaE}$ & & Insecticides & Li et al., 2017 [90] \\
\hline & $\mathrm{AChE}$ & & Insecticides & Qi et al., 2020 [59] \\
\hline & $\mathrm{AChE}$ & & Insecticides & Rabea et al., 2010 [49] \\
\hline & Octopamine, serotonin, dopamine & & Trace elements & Søvik et al., 2015 [72] \\
\hline & $\begin{array}{c}\text { Hyperresponsiveness, hyperactivity and } \\
\text { trembling }\end{array}$ & & Insecticides & Suchail et al., 2001 [101] \\
\hline & Protein level of synapsin & & Insecticides & Tavares et al., 2019 [102] \\
\hline & $\mathrm{AChE}$ & & Insecticides, acaricides & Weick and Thorn 2002 [79] \\
\hline & $\mathrm{AChE}$ & & Insecticide & Yao et al., 2018 [93] \\
\hline & Vtg expression & & Insecticides, Varroa destructor & Abbo et al., 2017 [47] \\
\hline & $\begin{array}{c}\text { Defensin 1, Abaecin, Hymenoptaecin } \\
\text { expressions }\end{array}$ & & Insecticides & Al Naggar et al., 2015 [77] \\
\hline & Nodulation & & $\begin{array}{c}\text { Dexamethasone (eicosanoid biosynthesis } \\
\text { inhibitor) }\end{array}$ & Bedick et al., 2001 [103] \\
\hline \multirow[t]{17}{*}{ Immunity } & $\begin{array}{c}\text { Hemocytes density, encapsulation } \\
\text { response and } \\
\text { antimicrobic activity }\end{array}$ & \multirow[t]{6}{*}{13} & Insecticides & Brandt et al., 2016 [104] \\
\hline & Lysozyme (LYS) and granulocytes count & & Fungicides, metals, EMS & Caliani et al., 2021 [43] \\
\hline & Immune response genes expression & & Insecticides, acaricides & Chaimanee et al., 2016 [75] \\
\hline & Vtg gene expression & & Insecticides & Christen et al., 2019 [105] \\
\hline & Vtg gene expression & & Insecticides & Christen et al., 2019 [86] \\
\hline & Phenoloxydase (PO) & & Gamma irradiation & Gagnaire et al., 2019 [98] \\
\hline & Immune genes expression & \multirow{21}{*}{17} & $\begin{array}{c}\text { Herbicides, fungicides, insecticides, } \\
\text { Varroa destructor }\end{array}$ & Gregorc et al., 2012 [87] \\
\hline & Immune gene expression & & Insecticides & Li et al., 2017 [90] \\
\hline & Vtg synthesis & & Insecticides & Pinto et al., 2000 [106] \\
\hline & Immune genes expression & & Insecticides & Zhu et al., 2020 [66] \\
\hline & Alkaline phosphatase (ALP) and GST & & $\begin{array}{l}\text { Insecticides, fungicides, herbicides and } \\
\text { mixture }\end{array}$ & Almasri et al., 2020 [83] \\
\hline & Alkaline phosphatase (ALP) and GST & & Insecticides & Badiou-Bénéteau et al., 2012 [53] \\
\hline & $\mathrm{Na}^{+}, \mathrm{K}^{+}-\mathrm{ATPase}$ assay & & Insecticides & Bendahou et al., 1999 [96] \\
\hline & ALP & & Insecticides & Bounias, 1985 [107] \\
\hline & ALP and GST & & Fungicides, metals, EMS & Caliani et al., 2021 [43] \\
\hline & ALP and GST & & Insecticides & Carvalho et al., 2013 [55] \\
\hline & $\begin{array}{l}\text { Genes encoding for enzymes involved in } \\
\text { phosphorylation }\end{array}$ & & Insecticides & Christen et al., 2019 [85] \\
\hline \multirow{10}{*}{ Metabolism } & Proteomic and metabolomic analysis & & Insecticides & du Rand et al., 2017 [82] \\
\hline & GST, CaEs and ALP & & Gamma irradiation & Gagnaire et al., 2019 [98] \\
\hline & GST and CaE & & Insecticides & Li et al., 2017 [90] \\
\hline & $\begin{array}{l}\text { Aspartate aminotransferase (AST), } \\
\text { alanine aminotransferase (ALT), ALP }\end{array}$ & & Insecticides & Paleolog et al., 2020 [108] \\
\hline & ATP assays and GADPH activity & & Mixtures & Prado et al., 2019 [50] \\
\hline & ATPase & & Insecticides & Rabea et al., 2010 [49] \\
\hline & GST, ALP & & Insecticides, Bacillus thurigiensis, mixtures & Renzi et al., 2016 [33] \\
\hline & Metabolic profile & & Insecticides & Shi et al., 2018 [109] \\
\hline & AST, ALT, ALP & & Acaricides & Strachecka et al., 2016 [110] \\
\hline & $\begin{array}{c}\text { Abundance of gut microbiota for } \\
\text { metabolic homeostasis, metabolic genes } \\
\text { expression }\end{array}$ & & Insecticides & Zhu et al., 2020 [66] \\
\hline
\end{tabular}


Table 2. Cont

\begin{tabular}{|c|c|c|c|c|}
\hline Endpoint & Test & $\mathbf{n}$ & Contaminants & Reference \\
\hline \multirow{9}{*}{$\begin{array}{l}\text { Oxidative } \\
\text { stress }\end{array}$} & GST, G6PDH & & $\begin{array}{l}\text { Insecticides, fungicides, herbicides and } \\
\text { mixture }\end{array}$ & Almasri et al., 2020 [83] \\
\hline & $\begin{array}{l}\text { GST, superoxide dismutase (SOD) and } \\
\text { catalase (CAT) genes expression }\end{array}$ & & $\mathrm{CdO}$ and $\mathrm{PbO}$ nanoparticles, mixtures & Al Naggar et al., 2020 [62] \\
\hline & polyphenol oxidase (PPO) & & Insecticides & Badawy et al., 2015 [84] \\
\hline & CAT & & Insecticides & Badiou-Bénéteau et al., 2012 [53 \\
\hline & CAT & & Insecticides & Carvalho et al., 2013 [55] \\
\hline & $\begin{array}{c}\text { CAT, SOD, glutathione peroxidase (GPx), } \\
\text { GST }\end{array}$ & & Gamma irradiation & Gagnaire et al., 2019 [98] \\
\hline & $\begin{array}{l}\alpha \text {-tocopherol and metallothionein-like } \\
\text { proteins (MTLPs) }\end{array}$ & 14 & Trace elements & Gauthier et al., 2016 [111] \\
\hline & $\begin{array}{c}\text { LPO, lutein, zeaxanthin, } \\
\alpha \text {-Cryptoxanthin, } \beta \text {-Cryptoxanthin, } \\
\beta \text {-Carotene, at-ROH, } \alpha \text {-Tocopherol }\end{array}$ & & Herbicides & Helmer et al., 2015 [64] \\
\hline & GST and PPO & & Insecticides & Li et al., 2017 [90] \\
\hline \multirow{11}{*}{$\begin{array}{l}\text { Genotoxicity } \\
\text { Primary stress } \\
\text { response }\end{array}$} & $\begin{array}{l}\text { SOD, CAT, reduced glutathione }(\mathrm{GSH}), \\
\text { protein thiol groups }(\mathrm{SH}), \\
\text { malondialdehyde (MDA) }\end{array}$ & & Trace elements & Nikolić et al., 2016 [112] \\
\hline & DNA methylation & & Insecticides & Paleolog et al., 2020 [108] \\
\hline & Peroxidase (POD), malondialdehyde & & Insecticides & Qi et al., 2020 [59] \\
\hline & GAPD, G6PD & & Insecticides, Bacillus thurigiensis, mixtures & Renzi et al., 2016 [33] \\
\hline & SOD, GPx, CAT, GST & & Acaricides & Strachecka et al., 2016 [110] \\
\hline & Nuclear abnormalities (NA) assay & 1 & Fungicides, metals, EMS & Caliani et al., 2021 [43] \\
\hline & HSP70 & 1 & Ethanol & Hranitz et al., 2010 [113] \\
\hline & Carbohydrates assay & 2 & $\begin{array}{l}\text { Insecticides } \\
\text { Insecticides } \\
\text { Herbicides }\end{array}$ & $\begin{array}{l}\text { Bendahou et al., } 1999 \text { [96] } \\
\text { Bounias, } 1985 \text { [107] } \\
\text { Helmer et al., } 2015 \text { [64] }\end{array}$ \\
\hline & Protein amount & 3 & Insecticides & Li et al., 2017 [90] \\
\hline & & & Insecticides & Pinto et al., 2000 [106] \\
\hline & Lipid amount & 1 & & Bounias, 1985 [107] \\
\hline
\end{tabular}

Table 3. Summary of semi-field studies divided by endpoint and contaminants.

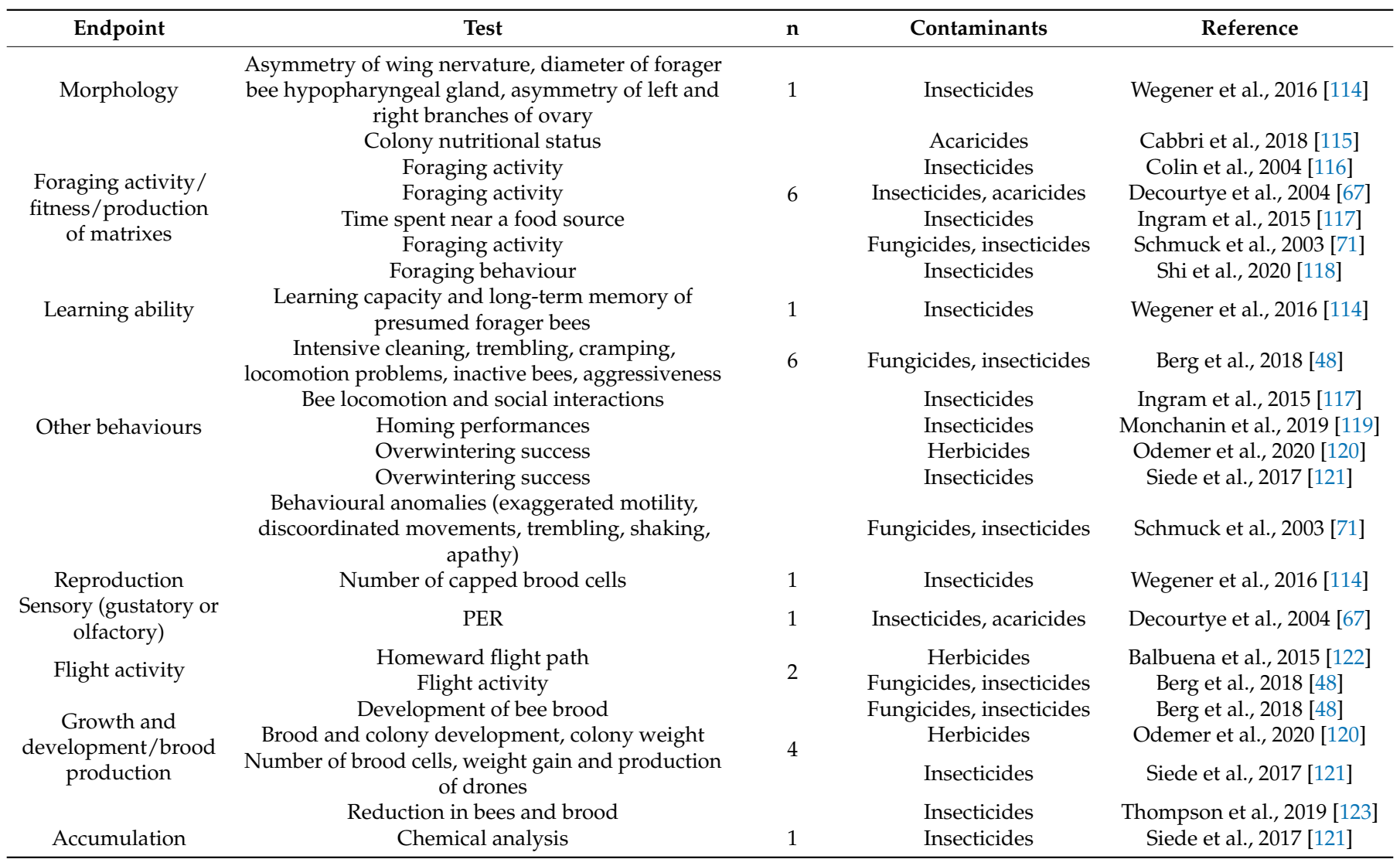


Table 4. Summary of semi-field studies divided by molecular and enzymatic endpoint and contaminants.

\begin{tabular}{|c|c|c|c|c|}
\hline Endpoint & Test & $\mathbf{n}$ & Contaminants & Reference \\
\hline \multirow[b]{2}{*}{ Detoxification } & GST & \multirow[b]{2}{*}{2} & Insecticides & Wegener et al., 2016 [114] \\
\hline & CYP450, CaEs, GST & & Insecticides & Zhu et al., 2020 [124] \\
\hline \multirow[t]{2}{*}{ Neurotoxicity } & Trembling and paralysis & 1 & Insecticides & Decourtye et al., 2004 [67] \\
\hline & Vtg and apolipophorin (APO) & & Acaricides & Cabbri et al., 2018 [115] \\
\hline \multirow[t]{2}{*}{ Immunity } & Hymenoptaecin gene expression & 3 & Insecticide & Siede et al., 2017 [121] \\
\hline & Vtg & & Insecticides & Wegener et al., 2016 [114] \\
\hline Metabolism & Phosphofructokinase & 1 & Insecticides & Wegener et al., 2016 [114] \\
\hline \multirow[t]{4}{*}{ Oxidative stress } & GST, phenoloxydase, glucose oxidase & 1 & Insecticides & Wegener et al., 2016 [114] \\
\hline & & & Acaricides & Cabbri et al., 2018 [115] \\
\hline & Protein amount & 3 & Insecticides & Wegener et al., 2016 [114] \\
\hline & & & Insecticides & Zhu et al., 2020 [124] \\
\hline
\end{tabular}

Table 5. Summary of field studies divided by endpoint and contaminants.

\begin{tabular}{|c|c|c|c|c|}
\hline Endpoint & Test & $\mathbf{n}$ & Contaminants & Reference \\
\hline \multirow[t]{9}{*}{ Morphology } & Wing asymmetry & 1 & Urbanisation & Leonard et al., 2018 [125] \\
\hline & Chemical analysis & & Metals & Al Naggar et al., 2013 [126] \\
\hline & Chemical analysis & & Insecticides & Al Naggar et al., 2015 [127] \\
\hline & Chemical analysis & & Insecticides & Al Naggar et al., 2015 [128] \\
\hline & Chemical analysis & & PAHs & Amorena et al., 2009 [129] \\
\hline & Chemical analysis & & Fungicides, insecticides & Amulen et al., 2017 [130] \\
\hline & Chemical analysis & & Insecticides & Codling et al., 2016 [131] \\
\hline & Chemical analysis & & Metals & Conti and Botrè, 2001 [132] \\
\hline & Chemical analysis & & Insecticides & El-Saad et al., 2017 [56] \\
\hline \multirow{10}{*}{ Accumulation } & Chemical analysis & & Herbicides, insecticides & Fulton et al., 2019 [133] \\
\hline & Chemical analysis & 18 & Metals & Kump et al., 1996 [134] \\
\hline & Chemical analysis & & Herbicides, fungicides, insecticides, acaricides & Mullin et al., 2010 [45] \\
\hline & Chemical analysis & & Trace elements & Nikolić et al., 2015 [135] \\
\hline & Chemical analysis & & PAHs & Perugini et al., 2009 [136] \\
\hline & Chemical analysis & & $\mathrm{SO}_{2}$ & Ponikvar et al., 2005 [137] \\
\hline & Chemical analysis & & Herbicides, fungicides, insecticides & Raimets et al., 2020 [46] \\
\hline & Chemical analysis & & Herbicides, insecticides, metals & Ruschioni et al., 2013 [138] \\
\hline & Gamma spectrometry & & Radiations & Tonelli et al., 1990 [139] \\
\hline & Chemical analysis & & Trace elements & van der Steen et al., 2012 [140] \\
\hline
\end{tabular}

Table 6. Summary of field studies divided by molecular and enzymatic endpoint and contaminants.

\begin{tabular}{|c|c|c|c|c|}
\hline Endpoint & Test & $\mathbf{n}$ & Contaminants & Reference \\
\hline \multirow{4}{*}{ Detoxification } & GST and metallothioneins (MT) & 4 & Trace elements & Badiou-Bénéteau et al., 2013 [54] \\
\hline & GST & & $\begin{array}{l}\text { Herbicides, fungicides, insecticides, } \\
\text { electromagnetic fields }\end{array}$ & Lupi et al., 2020 [44] \\
\hline & GST & & suspended dust and heavy metals & Nicewicz et al., 2020 [141] \\
\hline & $\begin{array}{c}\text { GST, esterases, epoxyde hydrolase and } \\
\text { DDT-dehydrochlorinase }\end{array}$ & & Insecticides & Yu et al., 1984 [142] \\
\hline \multirow{4}{*}{ Neurotoxicity } & AChE & 4 & Trace elements & Badiou-Bénéteau et al., 2013 [54] \\
\hline & $\mathrm{AChE}$ & & $\begin{array}{l}\text { Herbicides, fungicides, insecticides, } \\
\text { electromagnetic fields }\end{array}$ & Lupi et al., 2020 [44] \\
\hline & AChE & & suspended dust and heavy metals & Nicewicz et al., 2020 [141] \\
\hline & Esterases & & Insecticides & Yu et al., 1984 [142] \\
\hline Immunity & Defensin & 1 & suspended dust and heavy metals & Nicewicz et al., 2020 [141] \\
\hline \multirow{5}{*}{ Metabolism } & ALP and GST & 5 & Trace elements & Badiou-Bénéteau et al., 2013 [54] \\
\hline & ALP and Acidic phosphatase & & Trace elements & Bounias et al., 1996 [143] \\
\hline & ALP and GST & & $\begin{array}{l}\text { Herbicides, fungicides, insecticides, } \\
\text { electromagnetic fields }\end{array}$ & Lupi et al., 2020 [44] \\
\hline & GST & & suspended dust and heavy metals & Nicewicz et al., 2020 [141] \\
\hline & GST & & & Yu et al., 1984 [142] \\
\hline \multirow{4}{*}{ Oxidative stress } & SOD, CAT, GPx, GR & 4 & Insecticides & El-Saad et al., 2017 [56] \\
\hline & SOD and CAT & & Trace elements & Nikolić et al., 2015 [135] \\
\hline & CAT and GST & & $\begin{array}{l}\text { Herbicides, fungicides, insecticides, } \\
\text { electromagnetic fields }\end{array}$ & Lupi et al., $2020[44]$ \\
\hline & GST and total antioxidant capacity (TAC) & & suspended dust and heavy metals & Nicewicz et al., 2020 [141] \\
\hline Primary stress response & HSP70 & 1 & suspended dust and heavy metals & Nicewicz et al., 2020 [141] \\
\hline
\end{tabular}


Table 6 shows molecular endpoints examined in field studies. The effect that was studied with the highest degree of frequency concerned "detoxification" and "metabolism", both with 5 papers. The next two endpoints that were examined with a good degree of frequency were "neurotoxicity" and "oxidative stress"; the first was observed through the evaluation of AChE activity, the second mostly with the observation of CAT and SOD activity.

\section{Discussion}

The exposure of honey bees to environmental pollutants, especially agrochemical products, is causing a decline in their colonies [11,144], leading also to consequences for crop production, food security, and environmental health. For this reason, it is important to understand primarily both the benefits and the risks that the use of PPPs pose to the environment in order to make decisions about agricultural management. To determine the role of pesticides and other contaminants and their impact on honey bees it is essential to understand the kind of studies that have been conducted until now.

The majority of studies into the effects of pollutants on bees have been undertaken in North America and Europe, where important honey bee colony losses have been reported [14-16]. However, this phenomenon should be studied globally, in order to ascertain a better understanding of its causes. Although, PPPs tend to be most widely used in developed countries, they are increasingly being used in other parts of the world where regulations and best practices around their environmental impacts may not be as stringent [145].

The great majority of examined papers were about adult honey bees; it would be useful for there to be an improvement in the studies conducted related to other life stages, in order to have a better understanding of whether and how environmental contaminants may affect every stage of a honey bee's life cycle.

This review underlined that the majority of studies on honey bees are carried out in a laboratory more than in semi-field and field conditions, in a controlled environment and with controlled environmental exposure to the selected substances. The vast majority of papers about laboratory experiments reviewed focused on the sublethal effects, mostly about foraging activity, sensorial ability, neurotoxicity, detoxification, metabolism, and oxidative stress. In semi-field studies different responses both at macroscopic and microscopic levels were considered; however, in this review, only 14 papers of this kind were found. Honey bees, in the field, are exposed to multiple stressors and most of the field papers were monitoring studies where accumulation of various contaminants in Apis mellifera were investigated; only 8 papers $[28,33,50,57,62,71,83,95]$ analysed the sublethal effects of the contaminant mixtures on Apis mellifera. All these studies highlighted that honey bees are sensitive bioindicators of environmental pollution. Therefore, it is only through context monitoring that the honey bees decline should be examined, in order to understand its causes and to provide effective prevention tools to administrations.

In this review, it is highlighted that the most widely investigated PPPs are insecticides, because they were demonstrated to be harmful to non-target organisms, such as honey bees. Different authors observed that neonicotinoid insecticides, such as imidacloprid, thiamethoxam, acetamiprid, dinotefuran, thiacloprid, nitenpyram, and clothianidin, are able to damage honey bees olfactory learning performances [65,76,78], foraging activity $[65,68,69]$, and homing flight abilities [119]. This kind of compounds may cause neurotoxicity in honey bees, by altering AChEactivity which may be induced [97] or inhibited [84], and by modulating carboxylesterase (CaE) activity [53,82]. Furthermore, detoxification and antioxidant enzymes activities seem to be altered by neonicotinoids, such GST [53,81,82], CAT [53], PPO [84], ALP [53] and CYP450 [94] activities. Moreover, these compounds may affect the immune system for instance, by modulating the content of vitellogenin $[47,101]$, by reducing the hemocytes density, encapsulation response and antimicrobial activity [83], and by modulating the relative abundance of several key gut microbial molecules [66]. Several authors studied the effects of pyrethroid insecticides, 
such as deltamethrin, bifenthrin, cypermethrin, permethrin, and $\lambda$-cyhalotrin, on honey bees; these compounds seems to cause neurotoxicity by increasing AChE activity [59,91], modulating CaE activity [55]. Pyrethroids caused variations in lipid [107] and carbohydrates [96], reduced learning, memory performances [62,72] and foraging activity [67], and influenced bees locomotion and social interaction [117]. This class of insecticides is also able to cause variations in metabolic and detoxification activities, such as increasing GST activity [89,136], modulating ALP activity [107], inducing the expression of CYP450 monooxygenase [86], and inhibiting $\mathrm{Na}^{+}, \mathrm{K}^{+}$-ATPase activity [96]. Moreover, they may induce immune responses, cause changes in the activity of POD and in the content of MDA and LPO and induce oxidative stress [59]. Authors, who studied organophosphorus insecticides effects, observed an inhibition in the odour learning [79], a modulation of AChE activity $[75,77,84,95]$, a modulation of different immune system related genes and an induction of vitellogenin transcript [86]. El-Saad et al. (2017) [56] observed midguts ultrastructural modifications, a reduction of GSH levels, an inhibition of SOD, CAT and GPx activities, and an increase in MDA levels.

A recent review [146] underlined that other PPPs, such as fungicides and herbicides, that are not designed to target insects, may be factors that influence honey bees decline. For this reason, it would be important to increase the number of studies conducted related to their effects on these pollinators. Papers included in this review showed that the most frequently studied herbicide was glyphosate; it seems to cause a more indirect homing flight [122], to reduce sensitivity to sucrose and learning performance [69], to delay worker brood development [120], to have effects on the expression of CYP isoforms genes [87], and to slightly inhibit AChE activity [97].

Moreover, we believe that studies regarding other pollutants, such as PAHs and trace elements, should be improved, because of their presence in the environment that could cause honey bees exposure and adverse effects. Studies on trace elements underlined that pollutants, like aluminum, cadmium, selenium, lead, and copper, are able to influence foraging behavior $[63,70]$ and the development time [51,66], to cause histopathological alterations [57], to alter AChE, ALP, GST [43,54], CAT and SOD [107,140] activities. The European Food Safety Authority (EFSA) pointed out that the study of the impact of mixtures of chemicals also compared to non-chemical stressors, like Varroa destructor and viruses, on honey bee health are of great relevance, in view to support the implementation of a holistic risk assessment method $[147,148]$.

In field studies, it is more difficult to understand the effects caused by single contaminants, due to the presence of multiple stressors. Up to now, few papers have investigated the sublethal effects on honey bees in their natural conditions and habitats. Badiou-Bénéteau et al. (2013) [54] and Nikolić et al. (2015) [135] highlighted the presence of sublethal effects, characterized by oxidative stress and the induction of detoxification processes, in honey bees from more anthropized areas, due to the presence of neurotoxic pollutants, such as metals. Lupi et al. (2020) [44] observed that pesticide mixtures, characterized by the combination of fungicides, insecticides, and plant regulators, could cause an increase in Reactive Oxygen Species (ROS) that can inhibit AChE and CAT activities. An inhibition of some antioxidant stress biomarkers (GSH, SOD, CAT, GST) was also observed in specimens collected from anthropized areas [56]. Nicewicz et al. (2020) [141] observed the importance of defensin and HSP70 levels as indicators of urban multistress both at individual and colony levels. Further studies are needed to investigate the ecotoxicological status of honey bee colonies.

Another aspect to be pointed out is that in all three types of experimental conditions (laboratory, semi-filed and field), research studies have focused their attention on the development of some biomarkers to assess exposure to and the effects of contaminants on honey bees, such as esterases activity to evaluate neurotoxic effects, antioxidant enzymes activity, and predominantly CAT and SOD, together with detoxification reactions and metabolic activity. However, several responses, such as genotoxicity and immune system alteration, remain poorly explored and require an increased interest and a significant degree 
of effort to ensure that research studies are conducted. Colin et al. (2004) [116] observed, for example, that the suppression of the immune system may lead to a decrease in the individual performance and consequently in the population dynamics and the degree of disorders present in the colony. Moreover, Lazarov and Zhelyazkova (2019) [149] observed that Varroa destructor infestations are responsible for the weakening of honey bees' immune system, which may lead to a pronounced susceptibility of honey bees to contaminant exposure. To the best of our knowledge, Caliani et al. (2021) [43] is the only study that has been conducted into genotoxicity and that has examined Apis mellifera; in this study, it was observed that there are not only compounds such as EMS, with known genotoxic effects; indeed, there are also $\mathrm{Cd}$ and fungicides that have effects on the presence of hemocytes nuclear abnormalities.

While we have investigated the range of research approaches that have been used to study potential effects of contaminants on honey bees and provided a summary of main investigated effects (Tables 1-6), a full evaluation of effects direction was beyond the scope of this research. As there are 106 papers included in this review it is clear that there is an increasing corpus of literature that examines the effects of a wide range of compounds on bees. Only when certain research gaps are addressed, may this area benefit from a meta-analysis in the future to establish a clearer picture of the magnitude and direction of each effect.

\section{Conclusions}

The current review highlighted that Apis mellifera biological responses to external stressors were studied mostly in Europe and North America; consequently, there is a notable need to increase monitoring in other regions. Insecticides are widely studied compounds compared to other PPPs, or other classes such as e PAHs and trace elements. Laboratory studies are useful in order to determine the effects of specific compounds; however, field studies should be implemented, in order to gain a better understanding of the ecotoxicological status of $A$. mellifera in relation to environmental contamination patterns. Through the observation of the different responses examined by the authors, several gaps have been identified that should be addressed, particularly within enzymatic and molecular responses, such as those regarding immune system and genotoxicity. The development of an integrated approach, supported by statistical models could be vital, in order to combine responses at different levels, from molecular ones to the organism and the population. This could be a valid tool to evaluate the impact of contamination on these organisms and to support monitoring strategies not only at a scientific level, but also at a regulatory one.

Author Contributions: Conceptualization, S.C., I.C., G.C., T.C.; formal analysis, A.D.N.; investigation, A.D.N.; data curation, A.D.N.; writing-original draft preparation, A.D.N., I.C., T.C.; writing-review and editing, S.C., G.C.; supervision, S.C., G.C. All authors have read and agreed to the published version of the manuscript.

Funding: This research received no external funding.

Institutional Review Board Statement: Not applicable.

Informed Consent Statement: Not applicable.

Data Availability Statement: Data sharing not applicable.

Conflicts of Interest: The authors declare no conflict of interest.

\section{References}

1. Kennedy, C.M.; Lonsdorf, E.; Neel, M.C.; Williams, N.M.; Ricketts, T.H.; Winfree, R.; Bommarco, R.; Brittain, C.; Burley, A.L.; Cariveau, D.; et al. A global quantitative synthesis of local and landscape effects on wild bee pollinators in agroecosystems. Ecol. Lett. 2013, 16, 584-599. [CrossRef] [PubMed]

2. Burkle, L.A.; Marlin, J.C.; Knight, T.M. Plant-Pollinator Interactions over 120 Years: Loss of Species, Co-Occurrence, and Function. Science 2013, 339, 1611-1615. [CrossRef] [PubMed] 
3. Potts, S.G.; Biesmeijer, J.C.; Kremen, C.; Neumann, P.; Schweiger, O.; Kunin, W.E. Global pollinator declines: Trends, impacts and drivers. Trends Ecol. Evol. 2010, 25, 345-353. [CrossRef]

4. vanEngelsdorp, D.; Meixner, M.D. A historical review of managed honey bee populations in Europe and the United States and the factors that may affect them. J. Invertebr. Pathol. 2010, 103, S80-S95. [CrossRef]

5. Klein, A.-M.; Vaissière, B.E.; Cane, J.H.; Steffan-Dewenter, I.; Cunningham, S.A.; Kremen, C.; Tscharntke, T. Importance of pollinators in changing landscapes for world crops. Proc. R. Soc. B Biol. Sci. 2007, 274, 303-313. [CrossRef]

6. Aguilar, R.; Ashworth, L.; Galetto, L.; Aizen, M.A. Plant reproductive susceptibility to habitat fragmentation: Review and synthesis through a meta-analysis. Ecol. Lett. 2006, 9, 968-980. [CrossRef] [PubMed]

7. Ashman, T.-L.; Knight, T.M.; Steets, J.A.; Amarasekare, P.; Burd, M.; Campbell, D.R.; Dudash, M.R.; Johnston, M.O.; Mazer, S.J.; Mitchell, R.J.; et al. Pollen limitation of plant reproduction: Ecological and evolutionary causes and consequences. Ecology 2004, 85, 2408-2421. [CrossRef]

8. de Groot, R.S.; Wilson, M.A.; Boumans, R.M.J. A typology for the classification, description and valuation of ecosystem functions, goods and services. Ecol. Econ. 2002, 41, 393-408. [CrossRef]

9. Gallai, N.; Salles, J.-M.; Settele, J.; Vaissière, B.E. Economic valuation of the vulnerability of world agriculture confronted with pollinator decline. Ecol. Econ. 2009, 68, 810-821. [CrossRef]

10. Formato, G.; Zilli, R.; Condoleo, R.; Marozzi, S.; Davis, I.; Smulders, F.J.M. Risk management in primary apicultural production. Part 2: A Hazard Analysis Critical Control Point approach to assuring the safety of unprocessed honey. Vet. Q. 2011, 31, 87-97. [CrossRef]

11. Biesmeijer, J.C. Parallel Declines in Pollinators and Insect-Pollinated Plants in Britain and the Netherlands. Science 2006, 313, 351-354. [CrossRef]

12. Cameron, S.A.; Lozier, J.D.; Strange, J.P.; Koch, J.B.; Cordes, N.; Solter, L.F.; Griswold, T.L. Patterns of widespread decline in North American bumble bees. Proc. Natl. Acad. Sci. USA 2011, 108, 662-667. [CrossRef] [PubMed]

13. Ollerton, J.; Erenler, H.; Edwards, M.; Crockett, R. Extinctions of aculeate pollinators in Britain and the role of large-scale agricultural changes. Science 2014, 346, 1360-1362. [CrossRef]

14. Jacques, A.; Laurent, M.; Ribiere-Chabert, M.; Saussac, M.; Bougeard, S.; Hendrikx, P.; Chauzat, M. Statistical analysis on the EPILOBEE dataset: Explanatory variables related to honeybee colony mortality in EU during a 2 year survey. EFSA Support. Publ. 2016, 13. [CrossRef]

15. Steinhauer, N.A.; Rennich, K.; Wilson, M.E.; Caron, D.M.; Lengerich, E.J.; Pettis, J.S.; Rose, R.; Skinner, J.A.; Tarpy, D.R.; Wilkes, J.T.; et al. A national survey of managed honey bee 2012-2013 annual colony losses in the USA: Results from the Bee Informed Partnership. J. Apic. Res. 2014, 53, 1-18. [CrossRef]

16. van der Zee, R.; Pisa, L.; Andonov, S.; Brodschneider, R.; Charrière, J.-D.; Chlebo, R.; Coffey, M.F.; Crailsheim, K.; Dahle, B.; Gajda, A.; et al. Managed honey bee colony losses in Canada, China, Europe, Israel and Turkey, for the winters of 2008-9 and 2009-10. J. Apic. Res. 2012, 51, 100-114. [CrossRef]

17. Carnesecchi, E.; Svendsen, C.; Lasagni, S.; Grech, A.; Quignot, N.; Amzal, B.; Toma, C.; Tosi, S.; Rortais, A.; Cortinas-Abrahantes, J.; et al. Investigating combined toxicity of binary mixtures in bees: Meta-analysis of laboratory tests, modelling, mechanistic basis and implications for risk assessment. Environ. Int. 2019, 133, 105256. [CrossRef] [PubMed]

18. Potts, S.G.; Imperatriz-Fonseca, V.L.; Ngo, H.T.; Biesmeijer, J.C.; Breeze, T.D.; Dicks, L.V.; Garibaldi, L.A.; Hill, R.; Settele, J.; Vanbergen, A.J. The Assessment Report on Pollinators, Pollination and Food Production: Summary for Policymakers; Secretariat for Intergovernmental Science-Policy Platform on Biodiversity and Ecosystem Services: Bonn, Germany, 2016; ISBN 978-92-807-3568-0.

19. Goulson, D.; Nicholls, E.; Botias, C.; Rotheray, E.L. Bee declines driven by combined stress from parasites, pesticides, and lack of flowers. Science 2015, 347, 1255957. [CrossRef] [PubMed]

20. European Food Safety Authority. Towards an integrated environmental risk assessment of multiple stressors on bees: Review of research projects in Europe, knowledge gaps and recommendations. EFSA J. 2014, 12, 3594.

21. Rortais, A.; Arnold, G.; Dorne, J.-L.; More, S.J.; Sperandio, G.; Streissl, F.; Szentes, C.; Verdonck, F. Risk assessment of pesticides and other stressors in bees: Principles, data gaps and perspectives from the European Food Safety Authority. Sci. Total Environ. 2017, 587-588, 524-537. [CrossRef]

22. Nazzi, F.; Pennacchio, F. Disentangling multiple interactions in the hive ecosystem. Trends Parasitol. 2014, 30, 556-561. [CrossRef]

23. Nazzi, F.; Brown, S.P.; Annoscia, D.; Del Piccolo, F.; Di Prisco, G.; Varricchio, P.; Della Vedova, G.; Cattonaro, F.; Caprio, E.; Pennacchio, F. Synergistic Parasite-Pathogen Interactions Mediated by Host Immunity Can Drive the Collapse of Honeybee Colonies. PLoS Pathog. 2012, 8, e1002735. [CrossRef]

24. Di Pasquale, G.; Alaux, C.; Le Conte, Y.; Odoux, J.-F.; Pioz, M.; Vaissière, B.E.; Belzunces, L.P.; Decourtye, A. Variations in the Availability of Pollen Resources Affect Honey Bee Health. PLoS ONE 2016, 11, e0162818. [CrossRef] [PubMed]

25. Conte, Y.L.; Navajas, M. Climate change: Impact on honey bee populations and diseases. Rev. Sci. Tech. Off. Int. Epizoot. 2008, $27,499-510$.

26. Tosi, S.; Nieh, J.C.; Sgolastra, F.; Cabbri, R.; Medrzycki, P. Neonicotinoid pesticides and nutritional stress synergistically reduce survival in honey bees. Proc. R. Soc. B Biol. Sci. 2017, 284, 20171711. [CrossRef]

27. Tong, L.; Nieh, J.C.; Tosi, S. Combined nutritional stress and a new systemic pesticide (flupyradifurone, Sivanto®) reduce bee survival, food consumption, flight success, and thermoregulation. Chemosphere 2019, 237, 124408. [CrossRef] [PubMed] 
28. Williamson, S.M.; Wright, G.A. Exposure to multiple cholinergic pesticides impairs olfactory learning and memory in honeybees. J. Exp. Biol. 2013, 216, 1799-1807. [CrossRef] [PubMed]

29. Klein, S.; Cabirol, A.; Devaud, J.-M.; Barron, A.B.; Lihoreau, M. Why Bees Are So Vulnerable to Environmental Stressors. Trends Ecol. Evol. 2017, 32, 268-278. [CrossRef] [PubMed]

30. Alaux, C.; Brunet, J.-L.; Dussaubat, C.; Mondet, F.; Tchamitchan, S.; Cousin, M.; Brillard, J.; Baldy, A.; Belzunces, L.P.; Le Conte, Y. Interactions between Nosema microspores and a neonicotinoid weaken honeybees (Apis mellifera). Environ. Microbiol. 2010, 12, 774-782. [CrossRef] [PubMed]

31. Vidau, C.; Diogon, M.; Aufauvre, J.; Fontbonne, R.; Viguès, B.; Brunet, J.-L.; Texier, C.; Biron, D.G.; Blot, N.; El Alaoui, H.; et al. Exposure to Sublethal Doses of Fipronil and Thiacloprid Highly Increases Mortality of Honeybees Previously Infected by Nosema ceranae. PLoS ONE 2011, 6, e21550. [CrossRef]

32. Pettis, J.S.; vanEngelsdorp, D.; Johnson, J.; Dively, G. Pesticide exposure in honey bees results in increased levels of the gut pathogen Nosema. Naturwissenschaften 2012, 99, 153-158. [CrossRef]

33. Renzi, M.T.; Amichot, M.; Pauron, D.; Tchamitchian, S.; Brunet, J.-L.; Kretzschmar, A.; Maini, S.; Belzunces, L.P. Chronic toxicity and physiological changes induced in the honey bee by the exposure to fipronil and Bacillus thuringiensis spores alone or combined. Ecotoxicol. Environ. Saf. 2016, 127, 205-213. [CrossRef]

34. Robinson, A.; Hesketh, H.; Lahive, E.; Horton, A.A.; Svendsen, C.; Rortais, A.; Dorne, J.L.; Baas, J.; Heard, M.S.; Spurgeon, D.J. Comparing bee species responses to chemical mixtures: Common response patterns? PLoS ONE 2017, 12, e0176289. [CrossRef] [PubMed]

35. Han, W.; Yang, Y.; Gao, J.; Zhao, D.; Ren, C.; Wang, S.; Zhao, S.; Zhong, Y. Chronic toxicity and biochemical response of Apis cerana cerana (Hymenoptera: Apidae) exposed to acetamiprid and propiconazole alone or combined. Ecotoxicology 2019, 28, 399-411. [CrossRef] [PubMed]

36. Sanchez-Bayo, F.; Goka, K. Impacts of Pesticides on Honey Bees. In Beekeeping and Bee Conservation-Advances in Research; Chambo, E.D., Ed.; InTech: Rijeca, Croatia, 2016; ISBN 978-953-51-2411-5.

37. Johnson, R.M. Honey Bee Toxicology. Annu. Rev. Entomol. 2015, 60, 415-434. [CrossRef]

38. Tosi, S.; Nieh, J.C. Lethal and sublethal synergistic effects of a new systemic pesticide, flupyradifurone (Sivanto ${ }^{\circledR}$ ), on honeybees. Proc. R. Soc. B Biol. Sci. 2019, 286, 20190433. [CrossRef] [PubMed]

39. Cooper, J.; Dobson, H. The benefits of pesticides to mankind and the environment. Crop Prot. 2007, 26, 1337-1348. [CrossRef]

40. Silva, V.; Mol, H.G.J.; Zomer, P.; Tienstra, M.; Ritsema, C.J.; Geissen, V. Pesticide residues in European agricultural soils-A hidden reality unfolded. Sci. Total Environ. 2019, 653, 1532-1545. [CrossRef] [PubMed]

41. Van Bruggen, A.H.C.; He, M.M.; Shin, K.; Mai, V.; Jeong, K.C.; Finckh, M.R.; Morris, J.G. Environmental and health effects of the herbicide glyphosate. Sci. Total Environ. 2018, 616-617, 255-268. [CrossRef]

42. Carvalho, F.P. Pesticides, environment, and food safety. Food Energy Secur. 2017, 6, 48-60. [CrossRef]

43. Caliani, I.; Campani, T.; Conti, B.; Cosci, F.; Bedini, S.; D’Agostino, A.; Ammendola, A.; Di Noi, A.; Gori, A.; Casini, S. Multibiomarker approach and IBR index to evaluate the effects of different contaminants on the ecotoxicological status of Apis mellifera. Ecotoxicol. Environ. Saf. 2021, 208, 111486. [CrossRef] [PubMed]

44. Lupi, D.; Tremolada, P.; Colombo, M.; Giacchini, R.; Benocci, R.; Parenti, P.; Parolini, M.; Zambon, G.; Vighi, M. Effects of Pesticides and Electromagnetic Fields on Honeybees: A Field Study Using Biomarkers. Int. J. Environ. Res. 2020, 14, 107-122. [CrossRef]

45. Mullin, C.A.; Frazier, M.; Frazier, J.L.; Ashcraft, S.; Simonds, R.; vanEngelsdorp, D.; Pettis, J.S. High Levels of Miticides and Agrochemicals in North American Apiaries: Implications for Honey Bee Health. PLoS ONE 2010, 5, e9754. [CrossRef] [PubMed]

46. Raimets, R.; Bontšutšnaja, A.; Bartkevics, V.; Pugajeva, I.; Kaart, T.; Puusepp, L.; Pihlik, P.; Keres, I.; Viinalass, H.; Mänd, M.; et al. Pesticide residues in beehive matrices are dependent on collection time and matrix type but independent of proportion of foraged oilseed rape and agricultural land in foraging territory. Chemosphere 2020, 238, 124555. [CrossRef]

47. Abbo, P.M.; Kawasaki, J.K.; Hamilton, M.; Cook, S.C.; DeGrandi-Hoffman, G.; Li, W.F.; Liu, J.; Chen, Y.P. Effects of Imidacloprid and Varroa destructor on survival and health of European honey bees, Apis mellifera: Survival and health of European honey bees. Insect Sci. 2017, 24, 467-477. [CrossRef] [PubMed]

48. Berg, C.; Hill, M.; Bonetti, C.; Mitchell, G.C.; Sharma, B. The effects of iprodione fungicide on survival, behavior, and brood development of honeybees (Apis mellifera L.) after one foliar application during flowering on mustard: Effects of iprodione application on honeybees. Environ. Toxicol. Chem. 2018, 37, 3086-3094. [CrossRef] [PubMed]

49. Rabea, E.I.; Nasr, H.M.; Badawy, M.E.I. Toxic Effect and Biochemical Study of Chlorfluazuron, Oxymatrine, and Spinosad on Honey Bees (Apis mellifera). Arch. Environ. Contam. Toxicol. 2010, 58, 722-732. [CrossRef]

50. Prado, A.; Pioz, M.; Vidau, C.; Requier, F.; Jury, M.; Crauser, D.; Brunet, J.-L.; Le Conte, Y.; Alaux, C. Exposure to pollen-bound pesticide mixtures induces longer-lived but less efficient honey bees. Sci. Total Environ. 2019, 650, 1250-1260. [CrossRef]

51. Hladun, K.R.; Kaftanoglu, O.; Parker, D.R.; Tran, K.D.; Trumble, J.T. Effects of selenium on development, survival, and accumulation in the honeybee (Apis mellifera L.): Selenium's impact on survival in honeybees. Environ. Toxicol. Chem. 2013, 32, 2584-2592. [CrossRef]

52. Mixson, T.A.; Abramson, C.I.; Nolf, S.L.; Johnson, G.; Serrano, E.; Wells, H. Effect of GSM cellular phone radiation on the behavior of honey bees (Apis mellifera). Sci. Bee Cult. 2009, 1, 22-27. 
53. Badiou-Bénéteau, A.; Carvalho, S.M.; Brunet, J.-L.; Carvalho, G.A.; Buleté, A.; Giroud, B.; Belzunces, L.P. Development of biomarkers of exposure to xenobiotics in the honey bee Apis mellifera: Application to the systemic insecticide thiamethoxam. Ecotoxicol. Environ. Saf. 2012, 82, 22-31. [CrossRef]

54. Badiou-Bénéteau, A.; Benneveau, A.; Géret, F.; Delatte, H.; Becker, N.; Brunet, J.L.; Reynaud, B.; Belzunces, L.P. Honeybee biomarkers as promising tools to monitor environmental quality. Environ. Int. 2013, 60, 31-41. [CrossRef]

55. Carvalho, S.M.; Belzunces, L.P.; Carvalho, G.A.; Brunet, J.-L.; Badiou-Beneteau, A. Enzymatic biomarkers as tools to assess environmental quality: A case study of exposure of the honeybee Apis mellifera to insecticides: Biomarker responses in honeybees exposed to pesticides. Environ. Toxicol. Chem. 2013, 32, 2117-2124. [CrossRef]

56. El-Saad, A.M.A.; Kheirallah, D.A.; El-Samad, L.M. Biochemical and histological biomarkers in the midgut of Apis mellifera from polluted environment at Beheira Governorate, Egypt. Environ. Sci. Pollut. Res. 2017, 24, 3181-3193. [CrossRef]

57. Dabour, K.; Al Naggar, Y.; Masry, S.; Naiem, E.; Giesy, J.P. Cellular alterations in midgut cells of honey bee workers (Apis mellifera L.) exposed to sublethal concentrations of $\mathrm{CdO}$ or $\mathrm{PbO}$ nanoparticles or their binary mixture. Sci. Total Environ. 2019, 651, 1356-1367. [CrossRef] [PubMed]

58. Tomé, H.V.V.; Schmehl, D.R.; Wedde, A.E.; Godoy, R.S.M.; Ravaiano, S.V.; Guedes, R.N.C.; Martins, G.F.; Ellis, J.D. Frequently encountered pesticides can cause multiple disorders in developing worker honey bees. Environ. Pollut. 2020, 256, 113420. [CrossRef] [PubMed]

59. Qi, S.; Niu, X.; hui Wang, D.; Wang, C.; Zhu, L.; Xue, X.; Zhang, Z.; Wu, L. Flumethrin at sublethal concentrations induces stresses in adult honey bees (Apis mellifera L.). Sci. Total Environ. 2020, 700, 134500. [CrossRef] [PubMed]

60. Arthidoro de Castro, M.B.; Martinez, L.C.; Cossolin, J.F.S.; Serra, R.S.; Serrão, J.E. Cytotoxic effects on the midgut, hypopharyngeal, glands and brain of Apis mellifera honey bee workers exposed to chronic concentrations of lambda-cyhalothrin. Chemosphere 2020, 248, 126075. [CrossRef]

61. Oliveira, C.R.; Domingues, C.E.C.; de Melo, N.F.S.; Roat, T.C.; Malaspina, O.; Jones-Costa, M.; Silva-Zacarin, E.C.M.; Fraceto, L.F. Nanopesticide based on botanical insecticide pyrethrum and its potential effects on honeybees. Chemosphere 2019, $236,124282$. [CrossRef]

62. AL Naggar, Y.; Dabour, K.; Masry, S.; Sadek, A.; Naiem, E.; Giesy, J.P. Sublethal effects of chronic exposure to CdO or PbO nanoparticles or their binary mixture on the honey bee (Apis mellifera L.). Environ. Sci. Pollut. Res. 2020, 27, 19004-19015. [CrossRef]

63. Decourtye, A.; Devillers, J.; Genecque, E.; Menach, K.L.; Budzinski, H.; Cluzeau, S.; Pham-Delegue, M.H. Comparative Sublethal Toxicity of Nine Pesticides on Olfactory Learning Performances of the Honeybee Apis mellifera. Arch. Environ. Contam. Toxicol. 2005, 48, 242-250. [CrossRef] [PubMed]

64. Helmer, S.H.; Kerbaol, A.; Aras, P.; Jumarie, C.; Boily, M. Effects of realistic doses of atrazine, metolachlor, and glyphosate on lipid peroxidation and diet-derived antioxidants in caged honey bees (Apis mellifera). Environ. Sci. Pollut. Res. 2015, 22, 8010-8021. [CrossRef] [PubMed]

65. Hladun, K.R.; Smith, B.H.; Mustard, J.A.; Morton, R.R.; Trumble, J.T. Selenium Toxicity to Honey Bee (Apis mellifera L.) Pollinators: Effects on Behaviors and Survival. PLoS ONE 2012, 7, e34137. [CrossRef]

66. Zhu, L.; Qi, S.; Xue, X.; Niu, X.; Wu, L. Nitenpyram disturbs gut microbiota and influences metabolic homeostasis and immunity in honey bee (Apis mellifera L.). Environ. Pollut. 2020, 258, 113671. [CrossRef]

67. Decourtye, A.; Devillers, J.; Cluzeau, S.; Charreton, M.; Pham-Delègue, M.-H. Effects of imidacloprid and deltamethrin on associative learning in honeybees under semi-field and laboratory conditions. Ecotoxicol. Environ. Saf. 2004, 57, 410-419. [CrossRef]

68. Hladun, K.R.; Di, N.; Liu, T.-X.; Trumble, J.T. Metal contaminant accumulation in the hive: Consequences for whole-colony health and brood production in the honey bee (Apis mellifera L.): Impact of metal contaminants on honey bee health. Environ. Toxicol. Chem. 2016, 35, 322-329. [CrossRef] [PubMed]

69. Herbert, L.T.; Vazquez, D.E.; Arenas, A.; Farina, W.M. Effects of field-realistic doses of glyphosate on honeybee appetitive behaviour. J. Exp. Biol. 2014, 217, 3457-3464. [CrossRef]

70. Morfin, N.; Goodwin, P.H.; Correa-Benitez, A.; Guzman-Novoa, E. Sublethal exposure to clothianidin during the larval stage causes long-term impairment of hygienic and foraging behaviours of honey bees. Apidologie 2019, 50, 595-605. [CrossRef]

71. Schmuck, R.; Stadler, T.; Schmidt, H.-W. Field relevance of a synergistic effect observed in the laboratory between an EBI fungicide and a chloronicotinyl insecticide in the honeybee (Apis mellifera L, Hymenoptera): Synergistic effect between fungicide and thiacloprid in honeybee. Pest Manag. Sci. 2003, 59, 279-286. [CrossRef]

72. Søvik, E.; Perry, C.J.; LaMora, A.; Barron, A.B.; Ben-Shahar, Y. Negative impact of manganese on honeybee foraging. Biol. Lett. 2015, 11, 20140989. [CrossRef]

73. Guez, D.; Zhu, H.; Zhang, S.W.; Srinivasan, M.V. Enhanced cholinergic transmission promotes recall in honeybees. J. Insect Physiol. 2010, 56, 1341-1348. [CrossRef]

74. Zhang, Z.Y.; Li, Z.; Huang, Q.; Zhang, X.W.; Ke, L.; Yan, W.Y.; Zhang, L.Z.; Zeng, Z.J. Deltamethrin Impairs Honeybees (Apis mellifera) Dancing Communication. Arch. Environ. Contam. Toxicol. 2020, 78, 117-123. [CrossRef]

75. Chaimanee, V.; Evans, J.D.; Chen, Y.; Jackson, C.; Pettis, J.S. Sperm viability and gene expression in honey bee queens (Apis mellifera) following exposure to the neonicotinoid insecticide imidacloprid and the organophosphate acaricide coumaphos. J. Insect Physiol. 2016, 89, 1-8. [CrossRef] 
76. Dai, P.-L.; Wang, Q.; Sun, J.-H.; Liu, F.; Wang, X.; Wu, Y.-Y.; Zhou, T. Effects of sublethal concentrations of bifenthrin and deltamethrin on fecundity, growth, and development of the honeybee Apis mellifera ligustica. Environ. Toxicol. Chem. 2010, 29, 644-649. [CrossRef] [PubMed]

77. Al Naggar, Y.; Wiseman, S.; Sun, J.; Cutler, G.C.; Aboul-Soud, M.; Naiem, E.; Mona, M.; Seif, A.; Giesy, J.P. Effects of environmentally-relevant mixtures of four common organophosphorus insecticides on the honey bee (Apis mellifera L.). J. Insect Physiol. 2015, 82, 85-91. [CrossRef]

78. Imran, M.; Sheikh, U.A.A.; Nasir, M.; Ghaffar, M.A.; Tamkeen, A.; Iqbal, M.A. Do neonicotinoid insecticides impaired olfactory learning behavior in Apis mellifera? Int. J. Ind. Entomol. 2019, 38, 1-5.

79. Weick, J.; Thorn, R.S. Effects of Acute Sublethal Exposure to Coumaphos or Diazinon on Acquisition and Discrimination of Odor Stimuli in the Honey Bee (Hymenoptera: Apidae). J. Econ. Entomol. 2002, 95, 227-236. [CrossRef] [PubMed]

80. Wright, G.A.; Softley, S.; Earnshaw, H. Low doses of neonicotinoid pesticides in food rewards impair short-term olfactory memory in foraging-age honeybees. Sci. Rep. 2015, 5, 15322. [CrossRef] [PubMed]

81. Yang, E.-C.; Chang, H.-C.; Wu, W.-Y.; Chen, Y.-W. Impaired Olfactory Associative Behavior of Honeybee Workers Due to Contamination of Imidacloprid in the Larval Stage. PLoS ONE 2012, 7, e49472. [CrossRef]

82. du Rand, E.E.; Human, H.; Smit, S.; Beukes, M.; Apostolides, Z.; Nicolson, S.W.; Pirk, C.W.W. Proteomic and metabolomic analysis reveals rapid and extensive nicotine detoxification ability in honey bee larvae. Insect Biochem. Mol. Biol. 2017, 82, 41-51. [CrossRef]

83. Almasri, H.; Tavares, D.A.; Pioz, M.; Sené, D.; Tchamitchian, S.; Cousin, M.; Brunet, J.-L.; Belzunces, L.P. Mixtures of an insecticide, a fungicide and a herbicide induce high toxicities and systemic physiological disturbances in winter Apis mellifera honey bees. Ecotoxicol. Environ. Saf. 2020, 203, 111013. [CrossRef]

84. Badawy, M.E.I.; Nasr, H.M.; Rabea, E.I. Toxicity and biochemical changes in the honey bee Apis mellifera exposed to four insecticides under laboratory conditions. Apidologie 2015, 46, 177-193. [CrossRef]

85. Christen, V.; Krebs, J.; Bünter, I.; Fent, K. Biopesticide spinosad induces transcriptional alterations in genes associated with energy production in honey bees (Apis mellifera) at sublethal concentrations. J. Hazard. Mater. 2019, 378, 120736. [CrossRef]

86. Christen, V.; Joho, Y.; Vogel, M.; Fent, K. Transcriptional and physiological effects of the pyrethroid deltamethrin and the organophosphate dimethoate in the brain of honey bees (Apis mellifera). Environ. Pollut. 2019, 244, 247-256. [CrossRef] [PubMed]

87. Gregorc, A.; Evans, J.D.; Scharf, M.; Ellis, J.D. Gene expression in honey bee (Apis mellifera) larvae exposed to pesticides and Varroa mites (Varroa destructor). J. Insect Physiol. 2012, 58, 1042-1049. [CrossRef] [PubMed]

88. Johnson, R.M.; Wen, Z.; Schuler, M.A.; Berenbaum, M.R. Mediation of Pyrethroid Insecticide Toxicity to Honey Bees (Hymenoptera: Apidae) by Cytochrome P450 Monooxygenases. J. Econ. Entomol. 2006, 99, 5. [CrossRef]

89. Johnson, R.M.; Pollock, H.S.; Berenbaum, M.R. Synergistic Interactions Between In-Hive Miticides in Apis mellifera. J. Econ. Entomol. 2009, 102, 474-479. [CrossRef]

90. Li, Z.; Li, M.; He, J.; Zhao, X.; Chaimanee, V.; Huang, W.-F.; Nie, H.; Zhao, Y.; Su, S. Differential physiological effects of neonicotinoid insecticides on honey bees: A comparison between Apis mellifera and Apis cerana. Pestic. Biochem. Physiol. 2017, 140, 1-8. [CrossRef]

91. Mao, W.; Schuler, M.A.; Berenbaum, M.R. CYP9Q-mediated detoxification of acaricides in the honey bee (Apis mellifera). Proc. Natl. Acad. Sci. USA 2011, 108, 12657-12662. [CrossRef] [PubMed]

92. Papadopoulos, A.I.; Polemitou, I.; Laifi, P.; Yiangou, A.; Tananaki, C. Glutathione S-transferase in the insect Apis mellifera macedonica Kinetic characteristics and effect of stress on the expression of GST isoenzymes in the adult worker bee. Comp. Biochem. Physiol. 2004, 139, 93-97.

93. Yao, J.; Zhu, Y.C.; Adamczyk, J. Responses of Honey Bees to Lethal and Sublethal Doses of Formulated Clothianidin Alone and Mixtures. J. Econ. Entomol. 2018, 111, 1517-1525. [CrossRef]

94. Zaworra, M.; Nauen, R. New approaches to old problems: Removal of phospholipase A2 results in highly active microsomal membranes from the honey bee, Apis mellifera. Pestic. Biochem. Physiol. 2019, 161, 68-76. [CrossRef]

95. Badiou, A.; Meled, M.; Belzunces, L.P. Honeybee Apis mellifera acetylcholinesterase-A biomarker to detect deltamethrin exposure. Ecotoxicol. Environ. Saf. 2008, 69, 246-253. [CrossRef] [PubMed]

96. Bendahou, N.; Bounias, M.; Fleche, C. Toxicity of Cypermethrin and Fenitrothion on the Hemolymph Carbohydrates, Head Acetylcholinesterase, and Thoracic Muscle Na+, K+-ATPase of Emerging Honeybees (Apis mellifera L.). Ecotoxicol. Environ. Saf. 1999, 44, 139-146. [CrossRef] [PubMed]

97. Boily, M.; Sarrasin, B.; DeBlois, C.; Aras, P.; Chagnon, M. Acetylcholinesterase in honey bees (Apis mellifera) exposed to neonicotinoids, atrazine and glyphosate: Laboratory and field experiments. Environ. Sci. Pollut. Res. 2013, 20, 5603-5614. [CrossRef]

98. Gagnaire, B.; Bonnet, M.; Tchamitchian, S.; Cavalié, I.; Della-Vedova, C.; Dubourg, N.; Adam-Guillermin, C.; Brunet, J.-L.; Belzunces, L.P. Physiological effects of gamma irradiation in the honeybee, Apis mellifera. Ecotoxicol. Environ. Saf. 2019, 174, 153-163. [CrossRef] [PubMed]

99. Glavan, G.; Kos, M.; Božič, J.; Drobne, D.; Sabotič, J.; Kokalj, A.J. Different response of acetylcholinesterases in salt- and detergent-soluble fractions of honeybee haemolymph, head and thorax after exposure to diazinon. Comp. Biochem. Physiol. Part $C$ Toxicol. Pharmacol. 2018, 205, 8-14. [CrossRef] [PubMed] 
100. Hashimoto, J.H.; Ruvolo-Takasusuki, M.C.C.; Arnaut de Toledo, V. Evaluation of the Use of the Inhibition Esterase Activity on Apis mellifera as Bioindicators of Insecticide Thiamethoxam Pesticide Residues. Sociobiology 2003, 42, $693-699$.

101. Suchail, S.; Guez, D.; Belzunces, L.P. Discrepancy between acute and chronic toxicity induced by imidacloprid and its metabolites in Apis mellifera. Environ. Toxicol. Chem. 2001, 20, 2482-2486. [CrossRef]

102. Tavares, D.A.; Roat, T.C.; Silva-Zacarin, E.C.M.; Nocelli, R.C.F.; Malaspina, O. Exposure to thiamethoxam during the larval phase affects synapsin levels in the brain of the honey bee. Ecotoxicol. Environ. Saf. 2019, 169, 523-528. [CrossRef]

103. Bedick, J.C.; Tunaz, H.; Nor Aliza, A.R.; Putnam, S.M.; Ellis, M.D.; Stanley, D.W. Eicosanoids act in nodulation reactions to bacterial infections in newly emerged adult honey bees, Apis mellifera, but not in older foragers. Comp. Biochem. Physiol. Part C Toxicol. Pharmacol. 2001, 130, 107-117. [CrossRef]

104. Brandt, A.; Gorenflo, A.; Siede, R.; Meixner, M.; Büchler, R. The neonicotinoids thiacloprid, imidacloprid, and clothianidin affect the immunocompetence of honey bees (Apis mellifera L.). J. Insect Physiol. 2016, 86, 40-47. [CrossRef]

105. Christen, V.; Vogel, M.S.; Hettich, T.; Fent, K. A Vitellogenin Antibody in Honey Bees (Apis mellifera): Characterization and Application as Potential Biomarker for Insecticide Exposure. Environ. Toxicol. Chem. 2019, 38, 1074-1083. [CrossRef] [PubMed]

106. Pinto, L.Z.; Bitondi, M.M.G.; Simões, Z.L.P. Inhibition of vitellogenin synthesis in Apis mellifera workers by a juvenile hormone analogue, pyriproxyfen. J. Insect Physiol. 2000, 46, 153-160. [CrossRef]

107. Bounias, M. Sublethal Effects of a Synthetic Pyrethroid, Deltamethrin, on the Glycemia, the Lipemia, and the Gut Alkaline Phosphatases of Honeybees. Pestic. Biochem. Phys. 1985, 24, 149-160. [CrossRef]

108. Paleolog, J.; Wilde, J.; Siuda, M.; Bąk, B.; Wójcik, Ł.; Strachecka, A. Imidacloprid markedly affects hemolymph proteolysis, biomarkers, DNA global methylation, and the cuticle proteolytic layer in western honeybees. Apidologie 2020, 51, 620-630. [CrossRef]

109. Shi, T.; Burton, S.; Wang, Y.; Xu, S.; Zhang, W.; Yu, L. Metabolomic analysis of honey bee, Apis mellifera L. response to thiacloprid. Pestic. Biochem. Physiol. 2018, 152, 17-23. [CrossRef]

110. Strachecka, A.; Olszewski, K.; Paleolog, J. Varroa treatment with bromfenvinphos markedly suppresses honeybee biochemical defence levels. Entomol. Exp. Appl. 2016, 160, 57-71. [CrossRef]

111. Gauthier, M.; Aras, P.; Jumarie, C.; Boily, M. Low dietary levels of Al, Pb and Cd may affect the non-enzymatic antioxidant capacity in caged honey bees (Apis mellifera). Chemosphere 2016, 144, 848-854. [CrossRef] [PubMed]

112. Nikolić, T.V.; Kojić, D.; Orčić, S.; Batinić, D.; Vukašinović, E.; Blagojević, D.P.; Purać, J. The impact of sublethal concentrations of $\mathrm{Cu}, \mathrm{Pb}$ and $\mathrm{Cd}$ on honey bee redox status, superoxide dismutase and catalase in laboratory conditions. Chemosphere 2016, 164, 98-105. [CrossRef] [PubMed]

113. Hranitz, J.M.; Abramson, C.I.; Carter, R.P. Ethanol increases HSP70 concentrations in honeybee (Apis mellifera L.) brain tissue. Alcohol 2010, 44, 275-282. [CrossRef] [PubMed]

114. Wegener, J. Secondary biomarkers of insecticide-induced stress of honey bee colonies and their relevance for overwintering strength. Ecotoxicol. Environ. Saf. 2016, 132, 379-389. [CrossRef]

115. Cabbri, R.; Ferlizza, E.; Nanetti, A.; Monari, E.; Andreani, G.; Galuppi, R.; Isani, G. Biomarkers of nutritional status in honeybee haemolymph: Effects of different biotechnical approaches for Varroa destructor treatment and wintering phase. Apidologie 2018, 49, 606-618. [CrossRef]

116. Colin, M.E.; Bonmatin, J.M.; Moineau, I.; Gaimon, C.; Brun, S.; Vermandere, J.P. A Method to Quantify and Analyze the Foraging Activity of Honey Bees: Relevance to the Sublethal Effects Induced by Systemic Insecticides. Arch. Environ. Contam. Toxicol. 2004, 47, 387-395. [CrossRef]

117. Ingram, E.M.; Augustin, J.; Ellis, M.D.; Siegfried, B.D. Evaluating sub-lethal effects of orchard-applied pyrethroids using video-tracking software to quantify honey bee behaviors. Chemosphere 2015, 135, 272-277. [CrossRef] [PubMed]

118. Shi, J.; Yang, H.; Yu, L.; Liao, C.; Liu, Y.; Jin, M.; Yan, W.; Wu, X.B. Sublethal acetamiprid doses negatively affect the lifespans and foraging behaviors of honey bee (Apis mellifera L.) workers. Sci. Total Environ. 2020, 738, 139924. [CrossRef] [PubMed]

119. Monchanin, C.; Henry, M.; Decourtye, A.; Dalmon, A.; Fortini, D.; Bœuf, E.; Dubuisson, L.; Aupinel, P.; Chevallereau, C.; Petit, J.; et al. Hazard of a neonicotinoid insecticide on the homing flight of the honeybee depends on climatic conditions and Varroa infestation. Chemosphere 2019, 224, 360-368. [CrossRef]

120. Odemer, R.; Alkassab, A.T.; Bischoff, G.; Frommberger, M.; Wernecke, A.; Wirtz, I.P.; Pistorius, J.; Odemer, F. Chronic High Glyphosate Exposure Delays Individual Worker Bee (Apis mellifera L.) Development under Field Conditions. Insects 2020, 11, 664. [CrossRef]

121. Siede, R.; Faust, L.; Meixner, M.D.; Maus, C.; Grünewald, B.; Büchler, R. Performance of honey bee colonies under a long-lasting dietary exposure to sublethal concentrations of the neonicotinoid insecticide thiacloprid: Testing thiacloprid on bee colonies in a field trial. Pest Manag. Sci. 2017, 73, 1334-1344. [CrossRef]

122. Balbuena, M.S.; Tison, L.; Hahn, M.-L.; Greggers, U.; Menzel, R.; Farina, W.M. Effects of sublethal doses of glyphosate on honeybee navigation. J. Exp. Biol. 2015, 218, 2799-2805. [CrossRef]

123. Thompson, H.; Overmyer, J.; Feken, M.; Ruddle, N.; Vaughan, S.; Scorgie, E.; Bocksch, S.; Hill, M. Thiamethoxam: Long-term effects following honey bee colony-level exposure and implications for risk assessment. Sci. Total Environ. 2019, 654, 60-71. [CrossRef] 
124. Zhu, Y.C.; Caren, J.; Reddy, G.V.P.; Li, W.; Yao, J. Effect of age on insecticide susceptibility and enzymatic activities of three detoxification enzymes and one invertase in honey bee workers (Apis mellifera). Comp. Biochem. Physiol. Part C Toxicol. Pharmacol. 2020, 238, 108844. [CrossRef] [PubMed]

125. Leonard, R.J.; Wat, K.K.Y.; McArthur, C.; Hochuli, D.F. Urbanisation and wing asymmetry in the western honey bee (Apis mellifera, Linnaeus 1758) at multiple scales. PeerJ 2018, 6, e5940. [CrossRef] [PubMed]

126. Naggar, Y.A.A.; Naiem, E.-S.A.; Seif, A.I.; Mona, M.H. Honey bees and their products as a bio-in- dicator of environmental pollution with heavy metals. Mellifera 2013, 13, 1-20.

127. ALNaggar, Y.; Vogt, A.; Codling, G.; Naiem, E.; Mona, M.; Seif, A.; Robertson, A.J.; Giesy, J.P. Exposure of honeybees (Apis mellifera) in Saskatchewan, Canada to organophosphorus insecticides. Apidologie 2015, 46, 667-678. [CrossRef]

128. Al Naggar, Y.; Codling, G.; Vogt, A.; Naiem, E.; Mona, M.; Seif, A.; Giesy, J.P. Organophosphorus insecticides in honey, pollen and bees (Apis mellifera L.) and their potential hazard to bee colonies in Egypt. Ecotoxicol. Environ. Saf. 2015, 114, 1-8. [CrossRef]

129. Amorena, M.; Visciano, P.; Giacomelli, A.; Marinelli, E.; Sabatini, A.G.; Medrzycki, P.; Oddo, L.P.; De Pace, F.M.; Belligoli, P.; Di Serafino, G.; et al. Monitoring of levels of polycyclic aromatic hydrocarbons in bees caught from beekeeping: Remark 1. Vet. Res. Commun. 2009, 33, 165-167. [CrossRef]

130. Amulen, D.R.; Spanoghe, P.; Houbraken, M.; Tamale, A.; de Graaf, D.C.; Cross, P.; Smagghe, G. Environmental contaminants of honeybee products in Uganda detected using LC-MS/MS and GC-ECD. PLoS ONE 2017, 12, e0178546. [CrossRef]

131. Codling, G.; Al Naggar, Y.; Giesy, J.P.; Robertson, A.J. Concentrations of neonicotinoid insecticides in honey, pollen and honey bees (Apis mellifera L.) in central Saskatchewan, Canada. Chemosphere 2016, 144, 2321-2328. [CrossRef]

132. Conti, M.E.; Botrè, F. Honeybees and Their Products as Potential Bioindicators of Heavy Metals Contamination. Environ. Monit. Assess. 2001, 69, 267-282. [CrossRef]

133. Fulton, C.A.; Huff Hartz, K.E.; Fell, R.D.; Brewster, C.C.; Reeve, J.D.; Lydy, M.J. An assessment of pesticide exposures and land use of honey bees in Virginia. Chemosphere 2019, 222, 489-493. [CrossRef] [PubMed]

134. Kump, P.; Nečemer, M.; Šnajder, J. Determination of trace elements in bee honey, pollen and tissue by total reflection and radioisotope X-ray fluorescence spectrometry. Spectrochim. Acta Part B At. Spectrosc. 1996, 51, 499-507. [CrossRef]

135. Nikolić, T.V.; Purać, J.; Orčić, S.; Kojić, D.; Vujanović, D.; Stanimirović, Z.; Gržetić, I.; Ilijević, K.; Šikoparija, B.; Blagojević, D.P. Environmental effects on superoxide dismutase and catalase activity and expression in honey bee: Environmental Effect on SOD and CAT in Honey Bee. Arch. Insect Biochem. Physiol. 2015, 90, 181-194. [CrossRef] [PubMed]

136. Perugini, M.; Di Serafino, G.; Giacomelli, A.; Medrzycki, P.; Sabatini, A.G.; Persano Oddo, L.; Marinelli, E.; Amorena, M. Monitoring of Polycyclic Aromatic Hydrocarbons in Bees (Apis mellifera) and Honey in Urban Areas and Wildlife Reserves. J. Agric. Food Chem. 2009, 57, 7440-7444. [CrossRef] [PubMed]

137. Ponikvar, M.; Šnajder, J.; Sedej, B. Honey as a bioindicator for environmental pollution with $\mathrm{SO}_{2}$. Apidologie 2005, 36, 403-409. [CrossRef]

138. Ruschioni, S.; Riolo, P.; Minuz, R.L.; Stefano, M.; Cannella, M.; Porrini, C.; Isidoro, N. Biomonitoring with Honeybees of Heavy Metals and Pesticides in Nature Reserves of the Marche Region (Italy). Biol. Trace Elem. Res. 2013, 154, 226-233. [CrossRef]

139. Tonelli, D.; Gattavecchia, E.; Ghini, S.; Porrini, C.; Celli, G.; Mercuri, A.M. Honey bees and their products as indicators of environmental radioactive pollution. J. Radioanal. Nucl. Chem. Artic. 1990, 141, 427-436. [CrossRef]

140. van der Steen, J.J.M.; de Kraker, J.; Grotenhuis, T. Spatial and temporal variation of metal concentrations in adult honeybees (Apis mellifera L.). Environ. Monit. Assess. 2012, 184, 4119-4126. [CrossRef]

141. Nicewicz, Ł.; Nicewicz, A.W.; Kafel, A.; Nakonieczny, M. Set of stress biomarkers as a practical tool in the assessment of multistress effect using honeybees from urban and rural areas as a model organism: A pilot study. Environ. Sci. Pollut. Res. 2020, 1-13. [CrossRef]

142. Yu, S.J.; Robinson, F.A.; Nation, J.L. Detoxication capacity in the honey bee, Apis mellifera L. Pestic. Biochem. Physiol. 1984, 22, 360-368. [CrossRef]

143. Bounias, M.; Kruk, I.; Nectoux, M.; Popeskovic, D. Toxicology of Cupric Salts on Honeybees. V. Gluconate and Sulfate Action on Gut Alkaline and Acid Phosphatases. Ecotoxicol. Environ. Saf. 1996, 35, 67-76. [CrossRef] [PubMed]

144. Grab, H.; Branstetter, M.G.; Amon, N.; Urban-Mead, K.R.; Park, M.G.; Gibbs, J.; Blitzer, E.J.; Poveda, K.; Loeb, G.; Danforth, B.N. Agriculturally dominated landscapes reduce bee phylogenetic diversity and pollination services. Science 2019, 363, 282-284. [CrossRef] [PubMed]

145. Schreinemachers, P.; Tipraqsa, P. Agricultural pesticides and land use intensification in high, middle and low income countries. Food Policy 2012, 37, 616-626. [CrossRef]

146. Cullen, M.G.; Thompson, L.J.; Carolan, J.C.; Stout, J.C.; Stanley, D.A. Fungicides, herbicides and bees: A systematic review of existing research and methods. PLoS ONE 2019, 14, e0225743. [CrossRef] [PubMed]

147. EFSA Panel on Animal Health and Welfare (AHAW) Assessing the health status of managed honeybee colonies (HEALTHY-B): A toolbox to facilitate harmonised data collection. EFSA J. 2016, 14, e04578. [CrossRef]

148. European Food Safety Authority. Specifications for field data collection contributing to honey bee model corroboration and verification. EFSA Support. Publ. 2017, 14, 1234E.

149. Lazarov, S.; Zhelyazkova, I. Content of Crude Protein in the Body and Total Protein and Lysozyme in the Haemolymph of Worker Bees (Apis mellifera L.) according to their infestation with Varroa destructor. Zhivotnovadni Nauki 2019, 56, 9-15. 